\title{
Supporting Information: Electrochemical Surface Treatment of Discontinuous Carbon Fibers
}

Ngon T. Tran, Brendan A. Patterson, Alec G. Kolodziejczyk, Vincent M. Wu, Daniel B. Knorr, Jr. *

\section{Table of Contents}

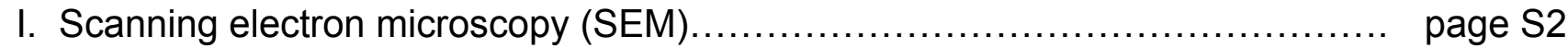

II. Fitting of XPS Data

a. Description of XPS Peak Assignments and Fitting

b. High Resolution XPS Spectra of Untreated Carbon Fibers.

c. High Resolution XPS Spectra of Carbon Fibers Treated with Ammonium Bicarbonate at Various Applied Voltages (applied voltage, $t_{\text {anode }}=10 \mathrm{~min}$., $\mathrm{T}_{i}$ $\left.\approx 23^{\circ} \mathrm{C}, 0.5 \mathrm{M} \mathrm{NH}_{4} \mathrm{HCO}_{3}\right)$.

d. High Resolution XPS Spectra of Carbon Fibers Treated with Ammonium Bicarbonate at Mixed Electrode Polarity $\left(16 \mathrm{~V}, t_{\text {anode }}=\mathrm{x}\right.$ followed by $t_{\text {cathode }}=$ 10 min. $-x, \mathrm{~T}_{i} \approx 23^{\circ} \mathrm{C}, 0.5 \mathrm{M} \mathrm{NH}_{4} \mathrm{HCO}_{3}$ ).

e. High Resolution XPS Spectra of Carbon Fibers Treated with Ammonium Bicarbonate at Various Initial Temperatures $\left(16 \mathrm{~V}, t_{\text {anode }}=5 \mathrm{~min}\right.$. followed by $t_{\text {cathode }}=5$ min., $\mathrm{T}_{i} \approx$ temp ${ }^{\circ} \mathrm{C}, 0.5 \mathrm{M} \mathrm{NH}_{4} \mathrm{HCO}_{3}$ ).

page S6

page S9

f. High Resolution XPS Spectra of Carbon Fibers Treated with Varying Ammonium Bicarbonate Concentrations (16 V, $t_{\text {anode }}=5 \mathrm{~min}$. followed by $t_{\text {cathode }}=5$ min., $\mathrm{T}_{i} \approx 23^{\circ} \mathrm{C}$, conc. $\mathrm{NH}_{4} \mathrm{HCO}_{3}$ ).

g. High Resolution XPS Spectra of Carbon Fibers Treated with Water and Different Electrolytes in the Mechanism Studies $\left(16 \mathrm{~V}, t_{\text {anode }}=10 \min ., \mathrm{T}_{i} \approx\right.$ $23{ }^{\circ} \mathrm{C}, 0.5 \mathrm{M}$ electrolyte)

III. References. 


\section{Scanning electron microscopy (SEM)}

Significant surface damage was observed for the prolonged electrochemical treatment of carbon fibers at $1.5 \mathrm{~V}\left(t_{\text {anode }}=2 \mathrm{~h}, \mathrm{~T}_{i} \approx 23{ }^{\circ} \mathrm{C}, 0.5 \mathrm{M}\right.$ ammonium bicarbonate $)$. In contrast, no surface damage was observed for the shorter electrochemical treatments ( $\leq 10 \mathrm{~min}$.) up to $18 \mathrm{~V}$. (See Figure 5 of manuscript.)
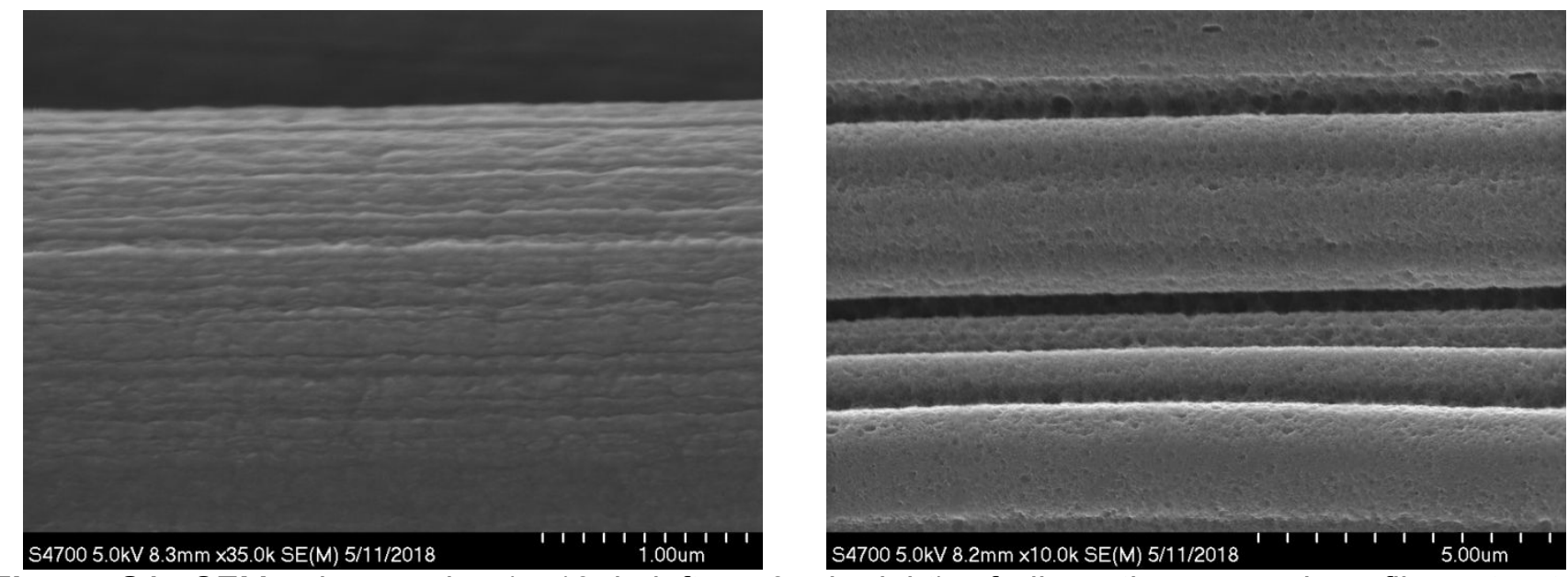

Figure S1. SEM micrographs (x $10 \mathrm{k}$ left, x $35 \mathrm{k}$ right) of discontinuous carbon fibers treated electrochemically with ammonium bicarbonate $\left(t_{\text {anode }}=2 \mathrm{~h}, \mathrm{~T}_{i} \approx 23^{\circ} \mathrm{C}, 0.5 \mathrm{M} \mathrm{NH}_{4} \mathrm{HCO}_{3}\right)$. 


\section{II a. Fitting of XPS Data}

XPS peak fitting was performed using the CasaXPS software following the procedure below. Table S1 provides the binding energies used in fitting individual species of $\mathrm{C}, \mathrm{N}, \mathrm{O}$, and $\mathrm{Cl}$ as well as literature precedent on similar use of these fits.

Table S1. XPS Peak Assignments and Binding Energies

\begin{tabular}{|l|c|c|c|}
\hline & Species & BE (eV) & References \\
\hline C 1s & $\mathrm{C}-\mathrm{C} / \mathrm{C}-\mathrm{H}$ & 284.6 & {$[1,2]$} \\
& $\mathrm{C}-\mathrm{O} / \mathrm{C}-\mathrm{N} / \mathrm{C}=\mathrm{N}$ & 286.0 & {$[2,4]$} \\
\cline { 2 - 4 } & $\mathrm{C}=\mathrm{O}$ & 287.1 & {$[1-4]$} \\
\cline { 2 - 4 } & $\mathrm{O}-\mathrm{C}=\mathrm{O}$ & 288.6 & {$[5]$} \\
\cline { 2 - 4 } $\mathrm{N} 1 \mathrm{~s}$ & $\pi \rightarrow \pi^{*}$ shake up & 281.1 & {$[3,4]$} \\
& aromatic N & 398.5 & {$[3,4,6]$} \\
& aliphatic $\mathrm{R}_{2} \mathrm{NH} / \mathrm{RNH}_{2}$ & 399.8 & {$[1,2,4,6-8]$} \\
& nitrile & 399.0 & {$[13,14]$} \\
& amide, lactam & 399.7 & {$[13,15]$} \\
& imide & 399.7 & {$[13]$} \\
& imine & 398.8 & {$[16]$} \\
& carbamate & 399.7 & {$[13]$} \\
& urea & 399.9 & {$[17]$} \\
& pyrrole & 400.5 & {$[11]$} \\
& pyridone & 400.8 & {$[12]$} \\
\hline & $\mathrm{C}-\mathrm{NH}_{3}{ }^{+}$ & 401.7 & {$[1,2,8][2,3,9]$} \\
\hline & pyridine N-oxide & $402.0-405.0$ & {$[12]$} \\
\hline $\mathrm{O} 1 \mathrm{~s}$ & nitrite & 404.1 & {$[13]$} \\
& $\mathrm{O}^{-\mathrm{C}}$ & 532.9 & {$[3,9]$} \\
\hline $\mathrm{Na} 1 \mathrm{~s}$ & $\mathrm{O}=\mathrm{C}$ & 531.3 & {$[3,10]$} \\
\hline $\mathrm{Cl} 1 \mathrm{~s}$ & $\mathrm{Na}^{+}$ & 1071.9 & {$[17]$} \\
\hline
\end{tabular}

$\underline{\mathrm{C}}$ 1s. The $\mathrm{C}-\mathrm{C} / \mathrm{C}-\mathrm{H}$ peak was fitted using a Hybrid Doniach Sunjic/GaussianLorentzian(product) asymmetric line shape using function call $H($ alpha, 50)G(84) in CasaXPS where alpha ranged from 0.03-0.06. The average alpha value for replicate analysis, which has a coefficient of variance that is less than $10 \%$, in each experiment is reported in Tables S2-S7. Peaks of $\mathrm{C} 1 \mathrm{~s}$, shown in Figures S2-14, were fit using peaks of equal FWHM ( 1.4-1.5 eV) for $\mathrm{C}=\mathrm{O}, \mathrm{C}-\mathrm{N} / \mathrm{C}-\mathrm{O} / \mathrm{C}=\mathrm{N}$, and $\mathrm{O}=\mathrm{C}-\mathrm{O} / \mathrm{O}=\mathrm{C}-\mathrm{N}$ based on best fits. Conversely, the FWHM of the $\mathrm{C}-\mathrm{C} / \mathrm{C}-\mathrm{H}$ peak and the $\Pi \rightarrow \pi^{*}$ shake-up peak were allowed to vary, but were close to 0.6-0.9 $\mathrm{eV}$ and $\sim 1-2 \mathrm{eV}$, respectively. The $\mathrm{C}-\mathrm{C} / \mathrm{C}-\mathrm{H}$ components of $\mathrm{C} 1 \mathrm{~s}$ peak was calibrated to a position at $284.6 \mathrm{eV}$, and the remaining carbons were constrained to the positions above the $\mathrm{C}-\mathrm{C} / \mathrm{C}-\mathrm{H}$ peak: by $1.4 \mathrm{eV}$ for $\mathrm{C}-\mathrm{N} / \mathrm{C}-\mathrm{O} / \mathrm{C}=\mathrm{N}$ peak, by $2.5 \mathrm{eV}$ for $\mathrm{C}=\mathrm{O}$, by 4.0 for $\mathrm{O}-\mathrm{C}=\mathrm{O} / \mathrm{O}=\mathrm{C}-\mathrm{N}$, and by $6.5 \mathrm{eV}$ for $\mathrm{m} \rightarrow \pi^{*}$ shake-up peak.

$\mathrm{O}$ 1s. Splitting the oxygen signals into $\mathrm{O}-\mathrm{C}$ and $\mathrm{O}=\mathrm{C}$ components was not performed because the $\mathrm{O} 1 \mathrm{~s}$ signals were largely symmetrical and we felt separating the fit into two peaks was 
unjustified. The FWHM values, which have coefficient of variance values that are less than 10 $\%$, are reported as an average of all replicate analysis for each experiment (Tables S2-S8).

$\mathrm{N}$ 1s. Spectra for $\mathrm{N}$ 1s are shown in Figures $\mathrm{S} 2-14$ and were fit using one peak, because the $\mathrm{N}$ $1 \mathrm{~s}$ signals were usually symmetrical and dividing the signal into components may give erroneous compositions. The only except was for fibers that were treated with ammonium hydroxide, which were clearly asymmetric and would have very large FWHM (>3.2 e V) if no splitting of signals was performed. For this exception, the peaks used to fit the data correspond to $\mathrm{R}_{2} \mathrm{NH} / \mathrm{RNH}_{2} / \mathrm{RCONH}_{2}$ and $\mathrm{RNH}_{3}{ }^{+} /$hydrogen bonded nitrogen at $\sim 399.8 \mathrm{eV}$, and $\sim 401.7 \mathrm{eV}$, respectively. The FWHM values, which have coefficient of variance values that are less than 10 $\%$, are reported as an average of all replicate analysis for each experiment (Tables S2-S8).

$\mathrm{Na} 1 s$ and $\mathrm{Cl} 2 p$. Because only a trace amount of the sodium and chlorine signals were observed, no fitting was performed. 


\section{II b. Fitting of XPS Data: High Resolution XPS Spectra of Untreated Carbon Fibers}
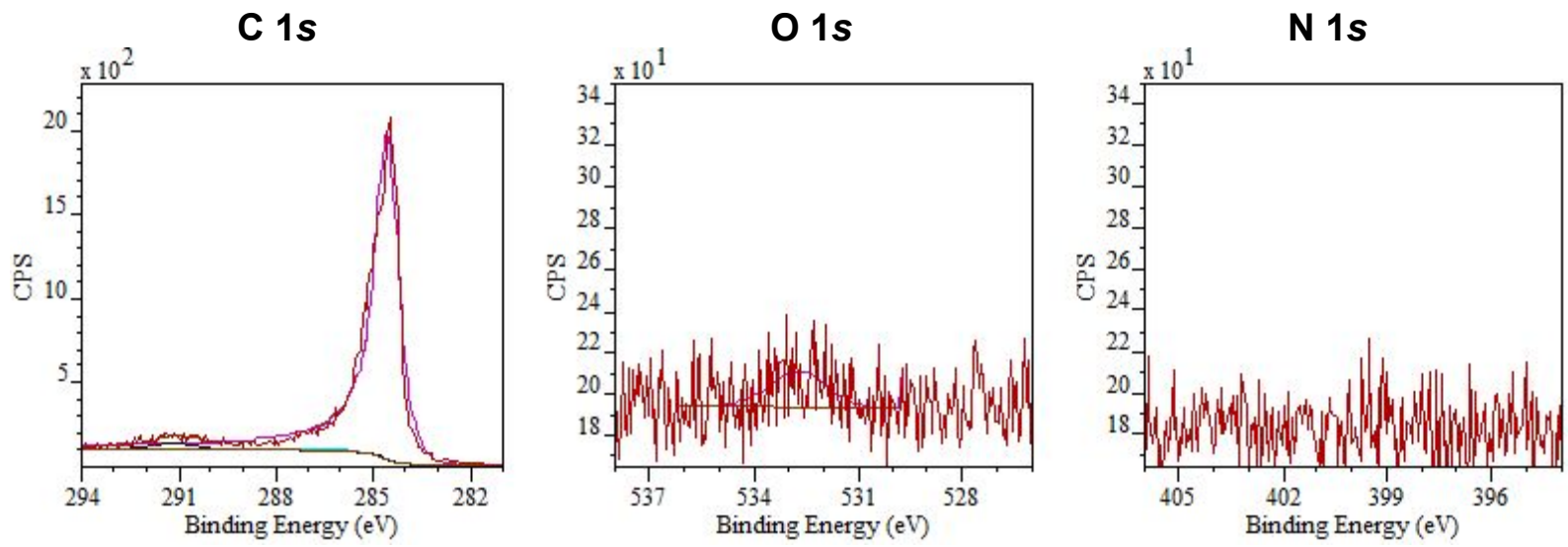

Figure S2. High resolution XPS spectra of $\mathrm{C} 1 \mathrm{~s}$ and $\mathrm{O} 1 \mathrm{~s}$ with individual fitting species for $25 \mathrm{~mm}$ untreated carbon fibers. (XPS spectra of $6 \mathrm{~mm}$ untreated carbon fibers are in Figure 3 of manuscript.) The $\mathrm{y}$-axis is in counts per second (CPS). (For $\mathrm{C}$ 1s spectra, magenta represents $\mathrm{C}-\mathrm{C} / \mathrm{C}-\mathrm{H}$, aqua blue represents $\mathrm{C}-\mathrm{O} / \mathrm{C}-\mathrm{N} / \mathrm{C}=\mathrm{N}$, red represents $\mathrm{C}=\mathrm{O}$, navy blue represents $\mathrm{O}-\mathrm{C}=\mathrm{O} / \mathrm{N}-\mathrm{C}=\mathrm{O}$ and black represents $\pi \rightarrow \pi^{*}$ shake-up peak.) Surface chemical compositions are reported in Table S2.

Table S2. Surface chemical compositions (at. \%) for untreated fibers based on high resolution XPS analyses. Binding energies (BE) and full width at half maximum (FWHM) are in $\mathrm{eV}$.

\begin{tabular}{|c|c|c|c|c|}
\hline \multirow[b]{2}{*}{ element } & \multicolumn{2}{|c|}{$6 \mathrm{~mm}$} & \multicolumn{2}{|c|}{$25 \mathrm{~mm}$} \\
\hline & $\begin{array}{c}\mathrm{BE} \\
(\mathrm{fwhm})\end{array}$ & at. $\%$ & $\begin{array}{c}\mathrm{BE} \\
(\mathrm{fwhm})\end{array}$ & at. $\%$ \\
\hline \multicolumn{5}{|l|}{ C $1 s$} \\
\hline $\mathrm{C}-\mathrm{C} / \mathrm{C}-\mathrm{H}$ & $\begin{array}{c}284.6 \\
(0.6)\end{array}$ & $95.0 \pm 1.5$ & $\begin{array}{c}284.6 \\
(0.7)\end{array}$ & $95.6 \pm 1.1$ \\
\hline $\mathrm{C}-\mathrm{O} / \mathrm{C}-\mathrm{N} / \mathrm{C}=\mathrm{N}$ & $\begin{array}{c}286.1 \\
(1.2)\end{array}$ & $1.4 \pm 0.1$ & $\begin{array}{c}286.0 \\
(1.5)\end{array}$ & $1.0 \pm 0.2$ \\
\hline $\mathrm{C}=\mathrm{O} / \mathrm{N}-\mathrm{C}-\mathrm{O}$ & --- & 0 & --- & 0 \\
\hline $\mathrm{O}-\mathrm{C}=\mathrm{O} / \mathrm{N}-\mathrm{C}=\mathrm{O}$ & --- & 0 & --- & 0 \\
\hline$\Pi \rightarrow \pi^{*}$ & $\begin{array}{c}290.9 \\
(1.8)\end{array}$ & $3.2 \pm 0.7$ & $\begin{array}{c}291.1 \\
(1.7)\end{array}$ & $3.4 \pm 0.9$ \\
\hline \multicolumn{5}{|l|}{$01 s$} \\
\hline $\mathrm{O}-\mathrm{C} / \mathrm{O}=\mathrm{C}$ & $\begin{array}{c}532.6 \\
(2.6)\end{array}$ & 100 & $\begin{array}{c}532.6 \\
(2.6)\end{array}$ & 100 \\
\hline \multicolumn{5}{|l|}{ N 1s } \\
\hline $\begin{array}{l}\mathrm{R}_{2} \mathrm{NH}_{2} / \mathrm{RNH}_{2} / \mathrm{RCONH}_{2} \\
\text { others }\end{array}$ & --- & 0 & --- & 0 \\
\hline $\begin{array}{l}\text { Alpha } \\
\text { T range }\left({ }^{\circ} \mathrm{C}\right)\end{array}$ & \multicolumn{2}{|c|}{$\begin{array}{c}0.032 \pm 0.004 \\
\text { N/A }\end{array}$} & \multicolumn{2}{|c|}{$\begin{array}{c}0.058 \pm 0.006 \\
\text { N/A }\end{array}$} \\
\hline
\end{tabular}




\section{II c. Fitting of XPS Data: High Resolution XPS Spectra of Carbon Fibers Treated with Ammonium Bicarbonate at Various Applied Voltages}

C 1 s
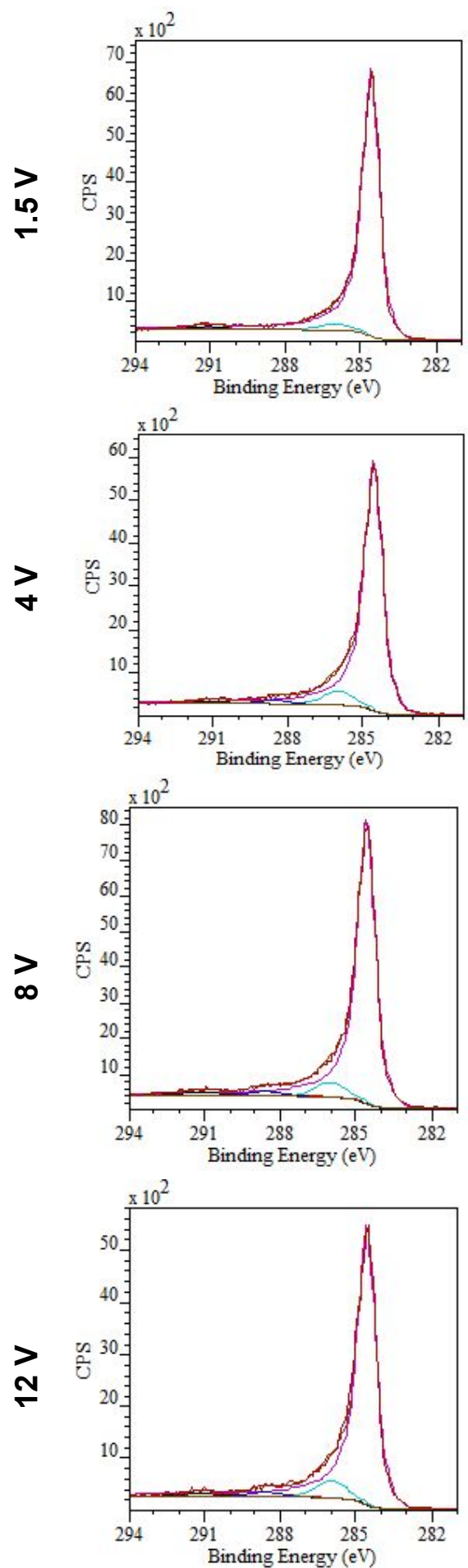

$01 s$
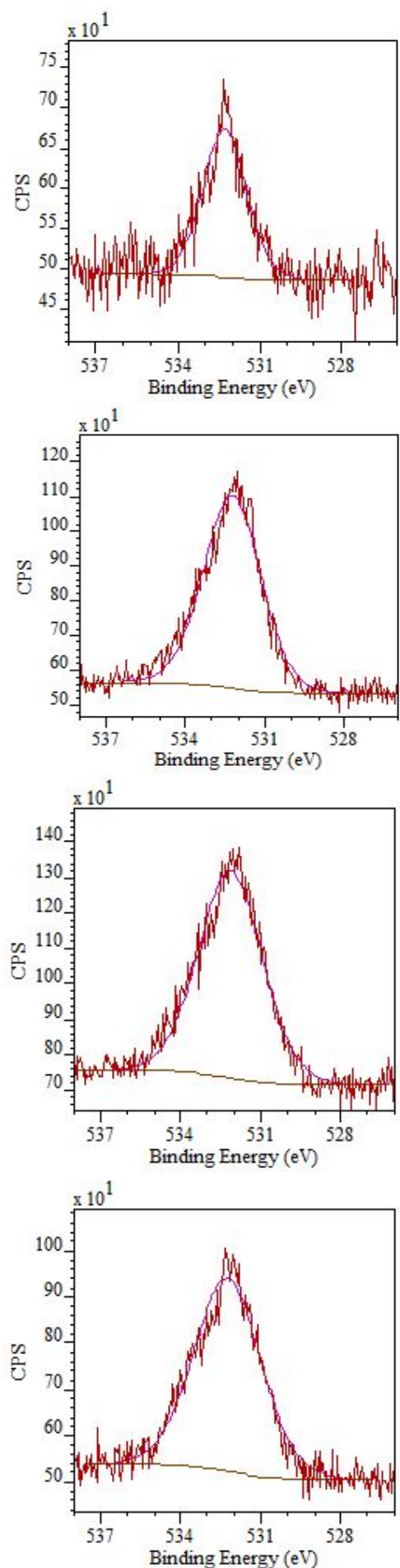

N $1 s$
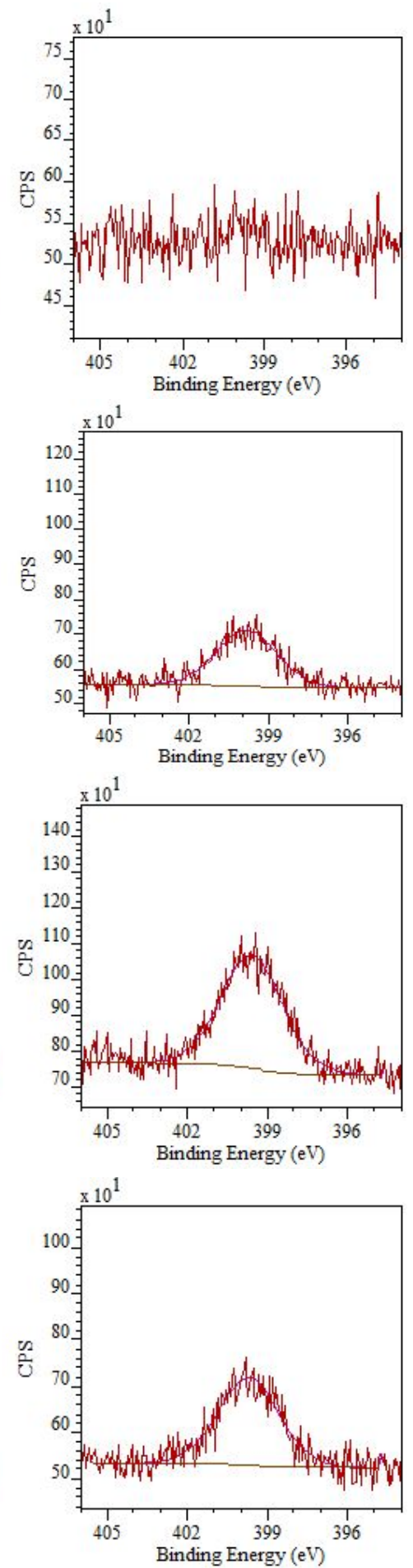

Figure S3. High resolution XPS spectra of $\mathrm{C} 1 \mathrm{~s}, \mathrm{O} 1 \mathrm{~s}$, and $\mathrm{N} 1 \mathrm{~s}$ with individual fitting species for carbon fibers treated at various applied voltages $\left(t_{\text {anode }}=10 \mathrm{~min} ., \mathrm{T}_{i} \approx 23{ }^{\circ} \mathrm{C}, 0.5 \mathrm{M}\right.$ ammonium bicarbonate). Spectra correspond to Figure 4 of manuscript. For $\mathrm{C} 1 s$ spectra, magenta represents $\mathrm{C}-\mathrm{C} / \mathrm{C}-\mathrm{H}$, aqua blue represents $\mathrm{C}-\mathrm{O} / \mathrm{C}-\mathrm{N} / \mathrm{C}=\mathrm{N}$, red represents $\mathrm{C}=\mathrm{O}$, navy blue represents $\mathrm{O}-\mathrm{C}=\mathrm{O} / \mathrm{N}-\mathrm{C}=\mathrm{O}$ and black represents $\pi \rightarrow \pi^{*}$ shake-up peak. Surface chemical compositions are reported in Table S3. 
C $1 s$
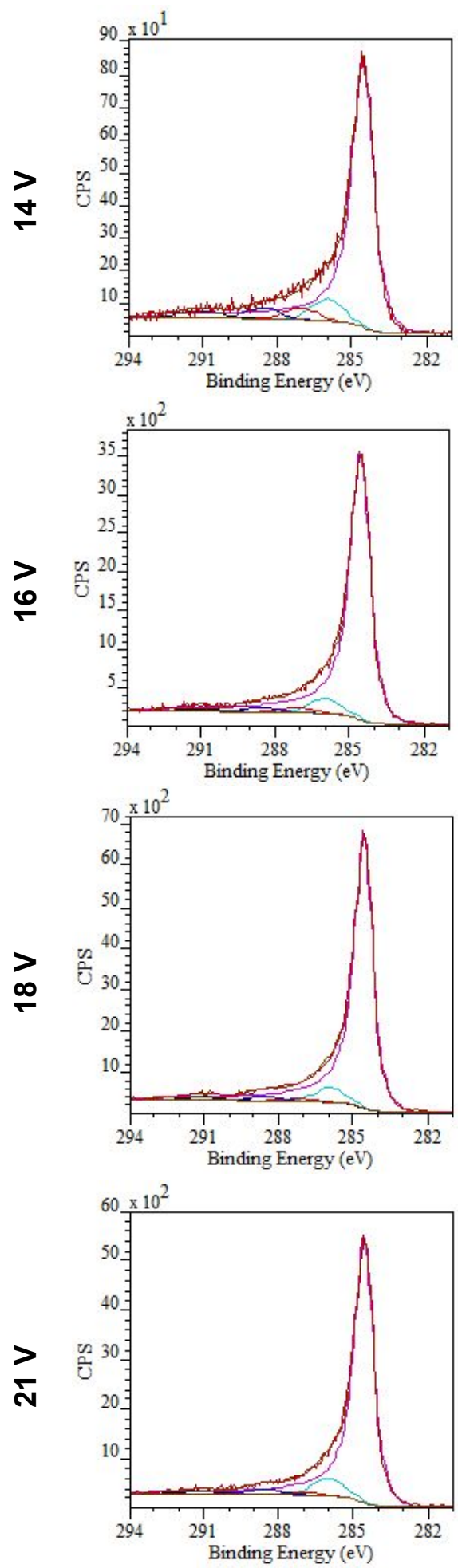

$01 s$
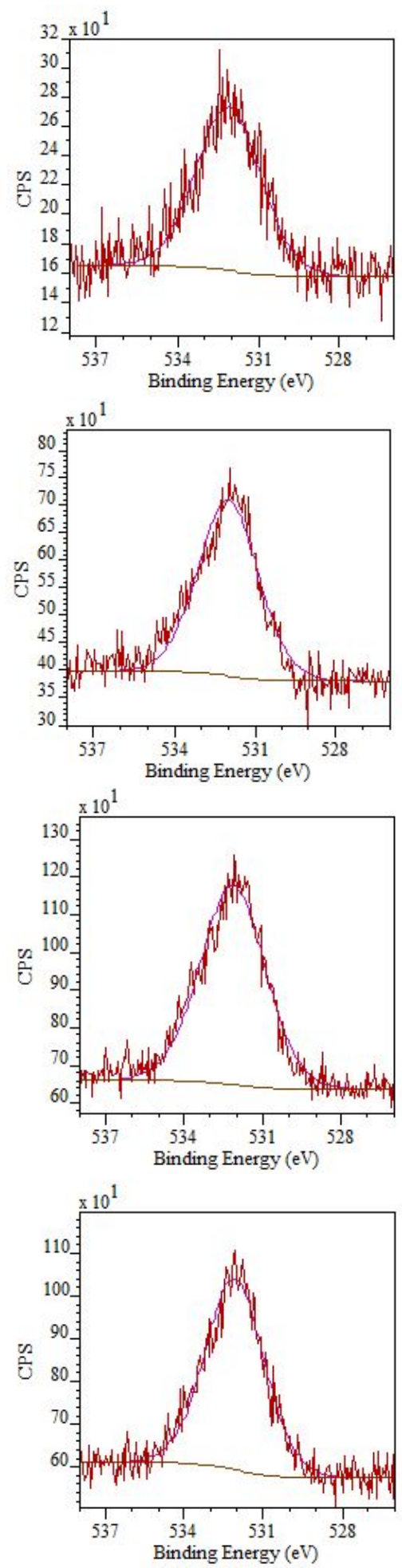

N $1 s$
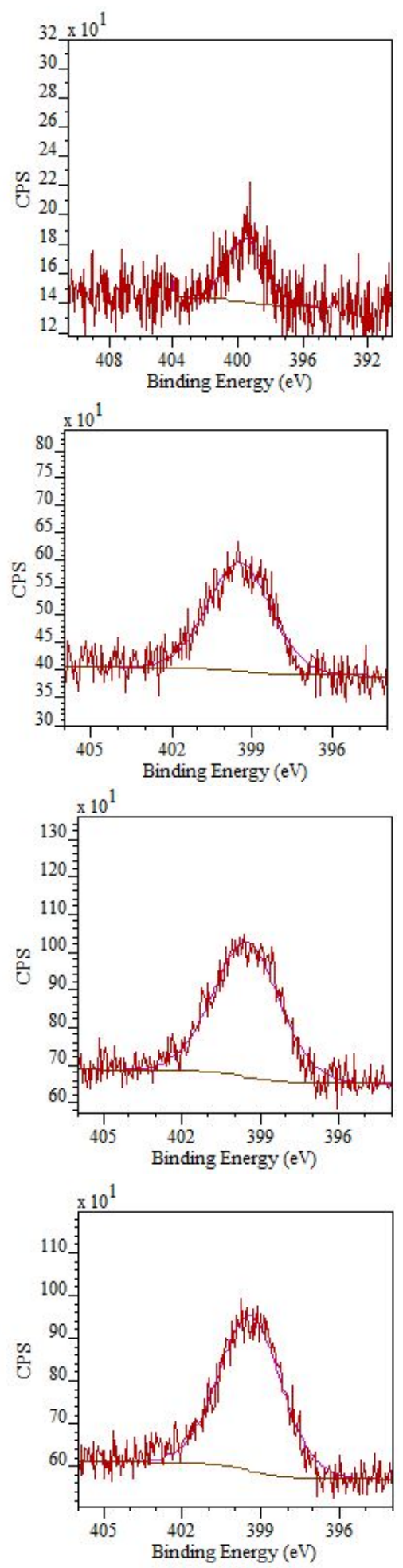

Figure S4. High resolution XPS spectra of $\mathrm{C} 1 s, \mathrm{O} 1 s$, and $\mathrm{N} 1 s$ with individual fitting species for carbon fibers treated at various applied voltages ( $t_{\text {anode }}=10 \mathrm{~min} ., \mathrm{T}_{i} \approx 23^{\circ} \mathrm{C}, 0.5 \mathrm{M}$ ammonium bicarbonate). Spectra correspond to Figure 4 of manuscript. For $\mathrm{C} 1 \mathrm{~s}$ spectra, magenta represents $\mathrm{C}-\mathrm{C} / \mathrm{C}-\mathrm{H}$, aqua blue represents $C-O / C-N / C=N$, red represents $C=O$, navy blue represents $O-C=O / N-C=O$ and black represents $\pi \rightarrow \pi^{*}$ shake-up peak.) Surface chemical compositions are reported in Table S4. 
Table S3. Surface chemical compositions (at. \%) for the electrolyte concentration studies (Figure 4 of manuscript) based on high resolution XPS analyses. Binding energies (BE) and full width at half maximum (FWHM) are in eV. (Varying applied voltage, $t_{\text {anode }}=10 \mathrm{~min}$., $\mathrm{T}_{i} \approx 23^{\circ} \mathrm{C}, 0.50 \mathrm{M}$ ammonium bicarbonate)

\begin{tabular}{|c|c|c|c|c|c|c|c|c|}
\hline \multirow[b]{2}{*}{ element } & \multicolumn{2}{|c|}{$1.5 \mathrm{~V}$} & \multicolumn{2}{|c|}{$4 \mathrm{~V}$} & \multicolumn{2}{|c|}{$8 \mathrm{~V}$} & \multicolumn{2}{|c|}{$12 \mathrm{~V}$} \\
\hline & $\begin{array}{c}\text { BE } \\
\text { (fwhm) }\end{array}$ & at. $\%$ & $\begin{array}{c}\text { BE } \\
\text { (fwhm) }\end{array}$ & at. $\%$ & $\begin{array}{c}\text { BE } \\
\text { (fwhm) }\end{array}$ & at. $\%$ & $\begin{array}{c}\text { BE } \\
\text { (fwhm) }\end{array}$ & at. $\%$ \\
\hline \multicolumn{9}{|l|}{ C $1 s$} \\
\hline $\mathrm{C}-\mathrm{C} / \mathrm{C}-\mathrm{H}$ & $\begin{array}{c}284.6 \\
(0.8)\end{array}$ & $95.6 \pm 0.7$ & $\begin{array}{c}284.6 \\
(0.8)\end{array}$ & $89.1 \pm 0.5$ & $\begin{array}{c}284.6 \\
(0.8)\end{array}$ & $87.8 \pm 0.9$ & $\begin{array}{c}284.6 \\
(0.8)\end{array}$ & $87.6 \pm 0.4$ \\
\hline $\mathrm{C}-\mathrm{O} / \mathrm{C}-\mathrm{N} / \mathrm{C}=\mathrm{N}$ & $\begin{array}{c}286.0 \\
(1.5)\end{array}$ & $2.7 \pm 0.6$ & $\begin{array}{c}286.0 \\
(1.5)\end{array}$ & $7.3 \pm 0.4$ & $\begin{array}{r}286.1 \\
(1.5)\end{array}$ & $7.5 \pm 0.2$ & $\begin{array}{c}286.0 \\
(1.5)\end{array}$ & $8.0 \pm 0.4$ \\
\hline $\mathrm{C}=\mathrm{O} / \mathrm{N}-\mathrm{C}-\mathrm{O}$ & $\begin{array}{c}287.1 \\
(1.5)\end{array}$ & $0.2 \pm 0.2$ & $\begin{array}{c}287.1 \\
(1.5)\end{array}$ & $0.2 \pm$ & $\begin{array}{c}287.1 \\
(1.5)\end{array}$ & $0.6 \pm 0.2$ & $\begin{array}{c}287.1 \\
(1.5)\end{array}$ & $0.2 \pm 0.2$ \\
\hline $\mathrm{O}-\mathrm{C}=\mathrm{O} / \mathrm{N}-\mathrm{C}=\mathrm{O}$ & $\begin{array}{c}288.6 \\
(1.5)\end{array}$ & 0 & $\begin{array}{c}288.6 \\
(1.5)\end{array}$ & $2.0 \pm 0.3$ & $\begin{array}{c}288.6 \\
(1.5)\end{array}$ & $2.4 \pm 0.2$ & $\begin{array}{c}288.6 \\
(1.5)\end{array}$ & $2.7 \pm 0.4$ \\
\hline$\Pi \rightarrow \Pi^{*}$ & $\begin{array}{c}291.1 \\
(1.3)\end{array}$ & $1.5 \pm 0.1$ & $\begin{array}{c}291.1 \\
(1.5)\end{array}$ & $1.4 \pm 0.3$ & $\begin{array}{c}291.1 \\
(1.5)\end{array}$ & $1.7 \pm 0.5$ & $\begin{array}{c}291.1 \\
(1.5)\end{array}$ & $1.5 \pm 0.2$ \\
\hline \multicolumn{9}{|l|}{$01 s$} \\
\hline $\mathrm{O}-\mathrm{C} / \mathrm{O}=\mathrm{C}$ & $\begin{array}{c}532.3 \\
(2.2)\end{array}$ & 100 & $\begin{array}{c}532.2 \\
(2.5)\end{array}$ & 100 & $\begin{array}{c}532.1 \\
(2.8)\end{array}$ & 100 & $\begin{array}{c}532.2 \\
(2.9)\end{array}$ & 100 \\
\hline \multicolumn{9}{|l|}{$\mathrm{N} 1 \mathrm{~s}$} \\
\hline $\mathrm{R}_{2} \mathrm{NH}_{2} / \mathrm{RNH}_{2} / \mathrm{RCONH}_{2}$ & (trace) & & $\begin{array}{c}399.8 \\
(2.4)\end{array}$ & 100 & $\begin{array}{c}399.6 \\
(2.6)\end{array}$ & 100 & $\begin{array}{c}399.7 \\
(2.6)\end{array}$ & 100 \\
\hline \multicolumn{9}{|l|}{ Others } \\
\hline $\begin{array}{l}\text { Alpha } \\
\text { T ranqe }\left({ }^{\circ} \mathrm{C}\right)\end{array}$ & $\begin{array}{r}0.03 \\
21.0(r\end{array}$ & $\begin{array}{l} \pm 0.003 \\
\text { change) }\end{array}$ & $\begin{array}{r}0.03 \\
20.0(r\end{array}$ & $\begin{array}{l}0.003 \\
\text { change) }\end{array}$ & $\begin{array}{r}0 . \\
21.0(r\end{array}$ & $\begin{array}{l}0 \pm 0 \\
\text { change) }\end{array}$ & $\begin{array}{r}0.03 \\
22 .\end{array}$ & $\begin{array}{l}.005 \\
29.5\end{array}$ \\
\hline
\end{tabular}

Table S4. Surface chemical compositions (at. \%) for the electrolyte concentration studies (Figure 4 of manuscript) based on high resolution XPS analyses. Binding energies (BE) and full width at half maximum (FWHM) are in eV. (Varying applied voltage, $t_{\text {anode }}=10 \mathrm{~min}$., $\mathrm{T}_{i} \approx 23^{\circ} \mathrm{C}, 0.50 \mathrm{M}$ ammonium bicarbonate)

\begin{tabular}{|c|c|c|c|c|c|c|c|c|}
\hline \multirow[b]{2}{*}{ element } & \multicolumn{2}{|c|}{$14 \mathrm{~V}$} & \multicolumn{2}{|c|}{$16 \mathrm{~V}$} & \multicolumn{2}{|c|}{$18 \mathrm{~V}$} & \multicolumn{2}{|c|}{$21 \mathrm{~V}$} \\
\hline & $\begin{array}{c}\text { BE } \\
\text { (fwhm) }\end{array}$ & at. $\%$ & $\begin{array}{c}\text { BE } \\
\text { (fwhm) }\end{array}$ & at. $\%$ & $\begin{array}{c}\mathrm{BE} \\
\text { (fwhm) }\end{array}$ & at. $\%$ & $\begin{array}{c}\text { BE } \\
\text { (fwhm) }\end{array}$ & at. $\%$ \\
\hline \multicolumn{9}{|l|}{ C $1 s$} \\
\hline $\mathrm{C}-\mathrm{C} / \mathrm{C}-\mathrm{H}$ & $\begin{array}{c}284.6 \\
(0.8)\end{array}$ & $87.5 \pm 1.6$ & $\begin{array}{c}284.6 \\
(0.9)\end{array}$ & $85.5 \pm 1.1$ & $\begin{array}{c}284.6 \\
(0.9)\end{array}$ & $86.6 \pm 1.0$ & $\begin{array}{c}284.6 \\
(0.8)\end{array}$ & $85.5 \pm 1.1$ \\
\hline $\mathrm{C}-\mathrm{O} / \mathrm{C}-\mathrm{N} / \mathrm{C}=\mathrm{N}$ & $\begin{array}{c}286.0 \\
(1.5)\end{array}$ & $8.4 \pm 0.5$ & $\begin{array}{c}286.0 \\
(1.5)\end{array}$ & $7.8 \pm 0.8$ & $\begin{array}{c}286.1 \\
(1.5)\end{array}$ & $8.0 \pm 1.1$ & $\begin{array}{c}286.0 \\
(1.5)\end{array}$ & $8.7 \pm 0.4$ \\
\hline $\mathrm{C}=\mathrm{O} / \mathrm{N}-\mathrm{C}-\mathrm{O}$ & $\begin{array}{c}287.1 \\
(1.5)\end{array}$ & $1.2 \pm 0.5$ & $\begin{array}{c}287.1 \\
(1.5)\end{array}$ & $2.3 \pm 0.3$ & $\begin{array}{c}287.1 \\
(1.5)\end{array}$ & $1.5 \pm 0.1$ & $\begin{array}{c}287.1 \\
(1.5)\end{array}$ & $1.7 \pm 0.3$ \\
\hline $\mathrm{O}-\mathrm{C}=\mathrm{O} / \mathrm{N}-\mathrm{C}=\mathrm{O}$ & $\begin{array}{c}288.6 \\
(1.5)\end{array}$ & $1.8 \pm 0.2$ & $\begin{array}{c}288.6 \\
(1.5)\end{array}$ & $2.7 \pm 0.4$ & $\begin{array}{l}288.6 \\
(1.5)\end{array}$ & $2.4 \pm 0.2$ & $\begin{array}{c}288.6 \\
(1.5)\end{array}$ & $2.5 \pm 0.5$ \\
\hline$\Pi \rightarrow \pi^{*}$ & $\begin{array}{c}291.1 \\
(1.5)\end{array}$ & $1.2 \pm 1.1$ & $\begin{array}{c}291.1 \\
(1.8)\end{array}$ & $1.8 \pm 0.2$ & $\begin{array}{l}291.1 \\
(1.5)\end{array}$ & $1.4 \pm 0.6$ & $\begin{array}{c}291.1 \\
(1.6)\end{array}$ & $1.6 \pm 0.1$ \\
\hline \multicolumn{9}{|l|}{$01 s$} \\
\hline $\mathrm{O}-\mathrm{C} / \mathrm{O}=\mathrm{C}$ & $\begin{array}{l}532.1 \\
(2.7)\end{array}$ & 100 & $\begin{array}{l}532.0 \\
(2.6)\end{array}$ & 100 & $\begin{array}{c}532.1 \\
(2.7)\end{array}$ & 100 & $\begin{array}{c}532.1 \\
(2.7)\end{array}$ & 100 \\
\hline \multicolumn{9}{|l|}{$\mathrm{N} 1 \mathrm{~s}$} \\
\hline $\mathrm{R}_{2} \mathrm{NH}_{2} / \mathrm{RNH}_{2} / \mathrm{RCONH}_{2}$ & $\begin{array}{c}399.6 \\
(2.7)\end{array}$ & 100 & $\begin{array}{l}399.5 \\
(2.7)\end{array}$ & 100 & $\begin{array}{c}399.5 \\
(2.8)\end{array}$ & 100 & $\begin{array}{c}399.4 \\
(2.8)\end{array}$ & 100 \\
\hline \multicolumn{9}{|l|}{ others } \\
\hline $\begin{array}{l}\text { Alpha } \\
\text { T ranqe }\left({ }^{\circ} \mathrm{C}\right)\end{array}$ & \multicolumn{2}{|c|}{$\begin{array}{c}0.047 \pm 0.003 \\
22.0-58.0\end{array}$} & \multicolumn{2}{|c|}{$\begin{array}{c}0.040 \pm 0 \\
22.0-73.0\end{array}$} & \multicolumn{2}{|c|}{$\begin{array}{c}0.041 \pm 0.006 \\
22.0-80.0\end{array}$} & \multicolumn{2}{|c|}{$\begin{array}{c}0.030 \pm 0 \\
19.5-84.0\end{array}$} \\
\hline
\end{tabular}


II d. Fitting of XPS Data: High Resolution XPS Spectra of Carbon Fibers Treated with Ammonium Bicarbonate at Mixed Electrode Polarity
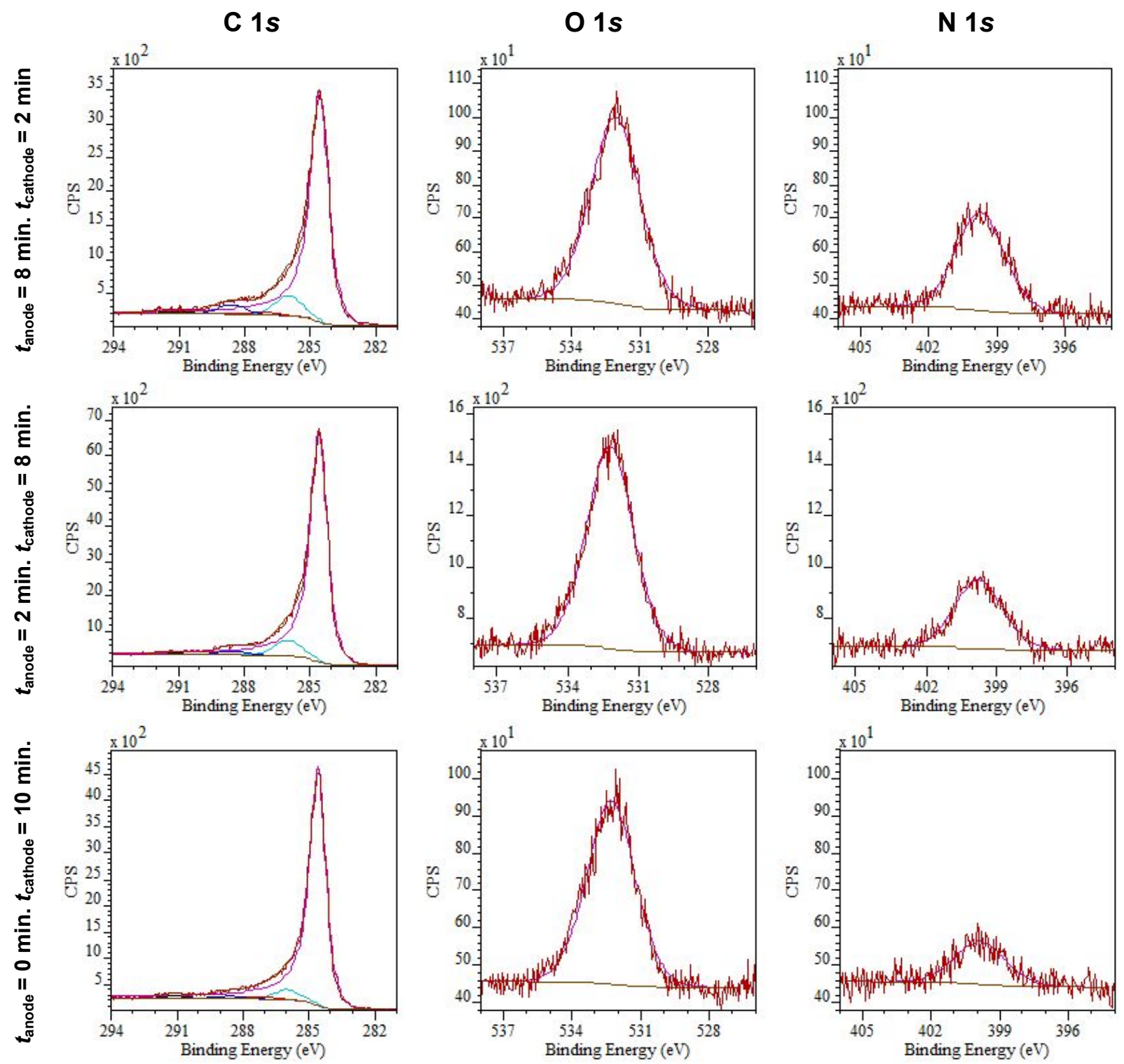

Figure S5. High resolution XPS spectra of $\mathrm{C} 1 \mathrm{~s}, \mathrm{O} 1 \mathrm{~s}$, and $\mathrm{N} 1 \mathrm{~s}$ with individual fitting species for carbon fibers treated at mixed electrode polarity $\left(16 \mathrm{~V}, \mathrm{~T}_{i} \approx 23{ }^{\circ} \mathrm{C}, 0.5 \mathrm{M} \mathrm{NH}_{4} \mathrm{HCO}_{3}\right)$, (Spectra corresponds to Figure 6 of manuscript and Table S5. For $\mathrm{C}$ 1s spectra, magenta represents $\mathrm{C}-\mathrm{C} / \mathrm{C}-\mathrm{H}$, aqua blue represents $\mathrm{C}-\mathrm{O} / \mathrm{C}-\mathrm{N} / \mathrm{C}=\mathrm{N}$, red represents $\mathrm{C}=\mathrm{O}$, navy blue represents $\mathrm{O}-\mathrm{C}=\mathrm{O} / \mathrm{N}-\mathrm{C}=\mathrm{O}$ and black represents $\pi \rightarrow \pi^{*}$ shake-up peak.) Surface chemical compositions at $t_{\text {anode }}=10$ min are reported in Figure S4 and Table S4. Surface chemical compositions at $t_{\text {anode }}=5 \mathrm{~min}$. followed by $t_{\text {cathode }}=5 \mathrm{~min}$. are reported in Figures S6 and Table S8. 
Table S5. Effect of electrode polarity on surface atomic concentrations of discontinuous fibers based on high resolution XPS analysis $\left(16 \mathrm{~V}, \mathrm{~T}_{i} \approx 23^{\circ} \mathrm{C}, 0.5 \mathrm{M} \mathrm{NH}_{4} \mathrm{HCO}_{3}\right)$.

\begin{tabular}{cccccc}
\hline Trials & $\begin{array}{c}\text { Time (min) } \\
\text { at Anode }\end{array}$ & $\begin{array}{c}\text { Time (min) } \\
\text { at Cathode }\end{array}$ & \%C & $\% \mathbf{O}$ & $\% \mathbf{N}$ \\
\hline 1 & 10 & 0 & $86.7 \pm 0.5$ & $6.5 \pm 0.4$ & $6.7 \pm 0.3$ \\
2 & 8 & 2 & $84.0 \pm 1.2$ & $8.9 \pm 0.8$ & $7.1 \pm 0.6$ \\
3 & 5 & 5 & $85.7 \pm 1.3$ & $9.4 \pm 0.9$ & $5.0 \pm 0.6$ \\
4 & 2 & 8 & $88.3 \pm 0.9$ & $7.3 \pm 0.9$ & $4.4 \pm 0.2$ \\
5 & 0 & 10 & $93.5 \pm 2.5$ & $5.4 \pm 1.8$ & $1.1 \pm 0.7$ \\
\hline
\end{tabular}

Table S6. Surface chemical compositions (at. \%) for the mixed-bias studies (Figure 6 of manuscript and Table S5) based on high resolution XPS analyses. Binding energies (BE) and full width at half maximum $(\mathrm{FWHM})$ are in eV. (Applied voltage $=16 \mathrm{~V}, t_{\text {anode }}=\mathrm{x}$ min. followed by $t_{\text {cathode }}=10-\mathrm{x} \min ., \mathrm{T}_{i} \approx 23^{\circ} \mathrm{C}, 0.5$ M ammonium bicarbonate)

\begin{tabular}{|c|c|c|c|c|c|c|}
\hline \multirow[b]{2}{*}{ element } & \multicolumn{2}{|c|}{$\begin{array}{c}t_{\text {anode }}=8 \mathrm{~min} . \\
t_{\text {cathode }}=2 \mathrm{~min} .\end{array}$} & \multicolumn{2}{|c|}{$\begin{array}{c}t_{\text {anode }}=2 \mathrm{~min} . \\
t_{\text {cathode }}=8 \mathrm{~min} .\end{array}$} & \multicolumn{2}{|c|}{$\begin{array}{c}t_{\text {anode }}=0 \mathrm{~min} . \\
t_{\text {cathode }}=10 \mathrm{~min} .\end{array}$} \\
\hline & $\begin{array}{c}\text { BE } \\
\text { (fwhm) }\end{array}$ & at. $\%$ & $\begin{array}{c}\text { BE } \\
\text { (fwhm) }\end{array}$ & at. $\%$ & $\begin{array}{c}\text { BE } \\
\text { (fwhm) }\end{array}$ & at. $\%$ \\
\hline \multicolumn{7}{|l|}{ C 1s } \\
\hline $\mathrm{C}-\mathrm{C} / \mathrm{C}-\mathrm{H}$ & $\begin{array}{c}284.6 \\
(0.9)\end{array}$ & $83.9 \pm 1.2$ & $\begin{array}{c}284.6 \\
(0.9)\end{array}$ & $87.6 \pm 1.1$ & $\begin{array}{l}284.6 \\
(0.8)\end{array}$ & $92.2 \pm 1.8$ \\
\hline $\mathrm{C}-\mathrm{O} / \mathrm{C}-\mathrm{N} / \mathrm{C}=\mathrm{N}$ & $\begin{array}{c}286.0 \\
(1.5)\end{array}$ & $10.0 \pm 0.6$ & $\begin{array}{c}286.0 \\
(1.5)\end{array}$ & $8.8 \pm 0.5$ & $\begin{array}{l}286.1 \\
(1.5)\end{array}$ & $4.9 \pm 1.4$ \\
\hline $\mathrm{C}=\mathrm{O} / \mathrm{N}-\mathrm{C}-\mathrm{O}$ & $\begin{array}{c}287.1 \\
(1.5)\end{array}$ & $1.2 \pm 0.5$ & $\begin{array}{l}287.1 \\
(1.5)\end{array}$ & 0 & $\begin{array}{l}287.1 \\
(1.5)\end{array}$ & $0.6 \pm 0.5$ \\
\hline $\mathrm{O}-\mathrm{C}=\mathrm{O} / \mathrm{N}-\mathrm{C}=\mathrm{O}$ & $\begin{array}{c}288.6 \\
(1.5)\end{array}$ & $3.9 \pm 0.7$ & $\begin{array}{c}288.6 \\
(1.5)\end{array}$ & $2.5 \pm 0.1$ & $\begin{array}{l}288.6 \\
(1.5)\end{array}$ & $0.9 \pm 0.5$ \\
\hline$\Pi \rightarrow \Pi^{*}$ & $\begin{array}{c}291.1 \\
(1.3)\end{array}$ & $0.9 \pm 0.2$ & $\begin{array}{c}291.1 \\
(1.5)\end{array}$ & $1.1 \pm 0.7$ & $\begin{array}{l}291.1 \\
(1.4)\end{array}$ & $1.4 \pm 0.7$ \\
\hline \multicolumn{7}{|l|}{$01 \mathrm{~s}$} \\
\hline $\mathrm{O}-\mathrm{C} / \mathrm{O}=\mathrm{C}$ & $\begin{array}{c}532.1 \\
(2.5)\end{array}$ & 100 & $\begin{array}{c}532.2 \\
(2.4)\end{array}$ & 100 & $\begin{array}{l}532.4 \\
(2.4)\end{array}$ & 100 \\
\hline \multicolumn{7}{|l|}{ N 1s } \\
\hline $\mathrm{R}_{2} \mathrm{NH}_{2} / \mathrm{RNH}_{2} / \mathrm{RCONH}_{2}$ & $\begin{array}{l}399.7 \\
(2.5)\end{array}$ & 100 & $\begin{array}{l}399.8 \\
(2.3)\end{array}$ & 100 & $\begin{array}{l}399.9 \\
(2.4)\end{array}$ & 100 \\
\hline \multicolumn{7}{|l|}{ others } \\
\hline $\begin{array}{l}\text { Alpha } \\
\text { T range }\left({ }^{\circ} \mathrm{C}\right)\end{array}$ & \multicolumn{2}{|c|}{$\begin{array}{c}0.035 \pm 0.005 \\
21.5-67.5\end{array}$} & \multicolumn{2}{|c|}{$\begin{array}{c}0.033 \pm 0.006 \\
22.0-67.0 \\
\end{array}$} & \multicolumn{2}{|c|}{$\begin{array}{c}0.041 \pm 0.006 \\
21.0-62.5 \\
\end{array}$} \\
\hline
\end{tabular}

aSurface chemical compositions at $t_{\text {anode }}=10 \mathrm{~min}$. are reported in Table S4, and those of $t_{\text {anode }}=5 \mathrm{~min}$. followed by $t_{\text {cathode }}=5 \mathrm{~min}$. are reported in Table S8. 
Table S7. Surface chemistry reproducibility assessment based on XPS analysis $\left(16 \mathrm{~V}, t_{\text {anode }}=5\right.$ min followed by $t_{\text {cathode }}=5 \mathrm{~min}, 0.5 \mathrm{M} \mathrm{NH}_{4} \mathrm{HCO}_{3}$ ). Experiments corresponds to those in Table S8.

\begin{tabular}{ccccc}
\hline Experiment & $\#^{\mathbf{a}}$ & $\mathbf{\% C}$ & $\mathbf{\% O}$ & $\mathbf{\% N}$ \\
\hline 1 & 3 & $84.4 \pm 0.6$ & $10.6 \pm 0.6$ & $4.9 \pm 0.1$ \\
2 & 3 & $85.4 \pm 1.3$ & $9.3 \pm 0.5$ & $5.3 \pm 0.9$ \\
3 & 3 & $87.1 \pm 2.3$ & $8.1 \pm 1.5$ & $4.7 \pm 0.9$ \\
4 & 12 & $85.7 \pm 0.9$ & $9.4 \pm 0.4$ & $5.0 \pm 0.5$ \\
Overall & 21 & $85.7 \pm 1.3$ & $9.4 \pm 0.9$ & $5.0 \pm 0.6$ \\
\hline
\end{tabular}

${ }^{a}$ Number of separate analyses of randomly chosen fibers

Table S8. Surface chemical speciations (at. \%) of C 1s regions for replicate runs (Table S7) based on high resolution XPS analyses. Binding energies (BE) and full width at half maximum (FWHM) are in eV. (Applied voltage $=16 \mathrm{~V}, t_{\text {anode }}=5 \mathrm{~min}$. followed by $t_{\text {cathode }}=5 \mathrm{~min} ., \mathrm{T}_{i} \approx 23{ }^{\circ} \mathrm{C}, 0.5 \mathrm{M}$ ammonium bicarbonate.)

\begin{tabular}{|c|c|c|c|c|c|c|c|c|}
\hline \multirow[b]{2}{*}{ element } & \multicolumn{2}{|c|}{ Experiment 1} & \multicolumn{2}{|c|}{ Experiment 2} & \multicolumn{2}{|c|}{ Experiment 3} & \multicolumn{2}{|c|}{ Experiment 4} \\
\hline & $\begin{array}{c}\text { BE } \\
\text { (fwhm) }\end{array}$ & at. $\%$ & $\begin{array}{c}\text { BE } \\
\text { (fwhm) }\end{array}$ & at. $\%$ & $\begin{array}{c}\text { BE } \\
\text { (fwhm) }\end{array}$ & at. $\%$ & $\begin{array}{c}\text { BE } \\
\text { (fwhm) }\end{array}$ & at. $\%$ \\
\hline \multicolumn{9}{|l|}{ C $1 s$} \\
\hline $\mathrm{C}-\mathrm{C} / \mathrm{C}-\mathrm{H}$ & $\begin{array}{c}284.6 \\
(0.9)\end{array}$ & $86.0 \pm 1.4$ & $\begin{array}{c}284.6 \\
(0.9)\end{array}$ & $85.8 \pm 0.4$ & $\begin{array}{c}284.6 \\
(0.9)\end{array}$ & $83.8 \pm 2.0$ & $\begin{array}{c}284.6 \\
(0.8)\end{array}$ & $84.9 \pm 1.9$ \\
\hline $\mathrm{C}-\mathrm{O} / \mathrm{C}-\mathrm{N} / \mathrm{C}=\mathrm{N}$ & $\begin{array}{r}286.0 \\
(1.5)\end{array}$ & $8.8 \pm 0.5$ & $\begin{array}{c}286.0 \\
(1.5)\end{array}$ & $8.5 \pm 0.5$ & $\begin{array}{c}286.0 \\
(1.5)\end{array}$ & $10.0 \pm 1.5$ & $\begin{array}{r}286.0 \\
(1.5)\end{array}$ & $10.5 \pm 0.8$ \\
\hline $\mathrm{C}=\mathrm{O} / \mathrm{N}-\mathrm{C}-\mathrm{O}$ & $\begin{array}{c}287.1 \\
(1.5)\end{array}$ & $1.1 \pm 0.3$ & $\begin{array}{c}287.1 \\
(1.5)\end{array}$ & $1.6 \pm 0.1$ & $\begin{array}{c}287.1 \\
(1.5)\end{array}$ & $1.3 \pm 0.2$ & $\begin{array}{c}287.1 \\
(1.5)\end{array}$ & $0.6 \pm 0.6$ \\
\hline $\mathrm{O}-\mathrm{C}=\mathrm{O} / \mathrm{N}-\mathrm{C}=\mathrm{O}$ & $\begin{array}{c}288.6 \\
(1.5)\end{array}$ & $3.4 \pm 0.5$ & $\begin{array}{c}288.6 \\
(1.5)\end{array}$ & $3.1 \pm 0.1$ & $\begin{array}{c}288.6 \\
(1.5)\end{array}$ & $3.2 \pm 0.8$ & $\begin{array}{c}288.6 \\
(1.5)\end{array}$ & $3.2 \pm 1.0$ \\
\hline$\Pi \rightarrow \Pi^{*}$ & $\begin{array}{r}291.1 \\
(1.4)\end{array}$ & $0.8 \pm 0.4$ & $\begin{array}{c}291.1 \\
(1.5)\end{array}$ & $1.0 \pm 0.7$ & $\begin{array}{c}291.1 \\
(1.4)\end{array}$ & $0.9 \pm 0.3$ & $\begin{array}{c}291.1 \\
(1.2)\end{array}$ & $0.8 \pm 0.7$ \\
\hline \multicolumn{9}{|l|}{$01 \mathrm{~s}$} \\
\hline $\mathrm{O}-\mathrm{C} / \mathrm{O}=\mathrm{C}$ & $\begin{array}{c}532.2 \\
(2.5)\end{array}$ & 100 & $\begin{array}{c}532.2 \\
(2.6)\end{array}$ & 100 & $\begin{array}{c}532.3 \\
(2.5)\end{array}$ & 100 & $\begin{array}{c}532.1 \\
(2.7)\end{array}$ & 100 \\
\hline \multicolumn{9}{|l|}{ N 1s } \\
\hline $\mathrm{R}_{2} \mathrm{NH}_{2} / \mathrm{RNH}_{2} / \mathrm{RCONH}_{2}$ & $\begin{array}{l}399.8 \\
(2.4)\end{array}$ & 100 & $\begin{array}{c}399.9 \\
(2.3)\end{array}$ & 100 & $\begin{array}{c}400.0 \\
(2.3)\end{array}$ & 100 & $\begin{array}{c}399.6 \\
(2.4)\end{array}$ & 100 \\
\hline \multicolumn{9}{|l|}{ others } \\
\hline Alpha & \multicolumn{2}{|c|}{$\begin{array}{c}0.040 \pm 0.002 \\
22.0-65.5\end{array}$} & \multicolumn{2}{|c|}{$\begin{array}{c}0.042 \pm 0.004 \\
22.0-66.0\end{array}$} & \multicolumn{2}{|c|}{$\begin{array}{c}0.041 \pm 0.004 \\
21.0-67.5\end{array}$} & \multicolumn{2}{|c|}{$\begin{array}{c}0.041 \pm 0.002 \\
21.0-67.0\end{array}$} \\
\hline
\end{tabular}


C $1 s$
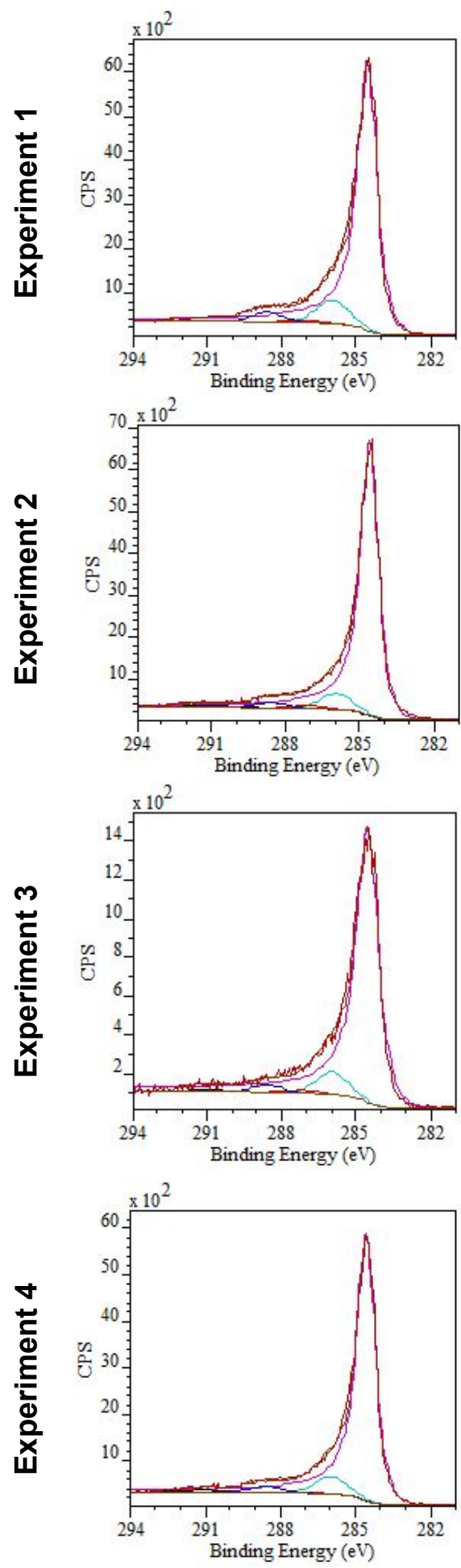

$01 s$
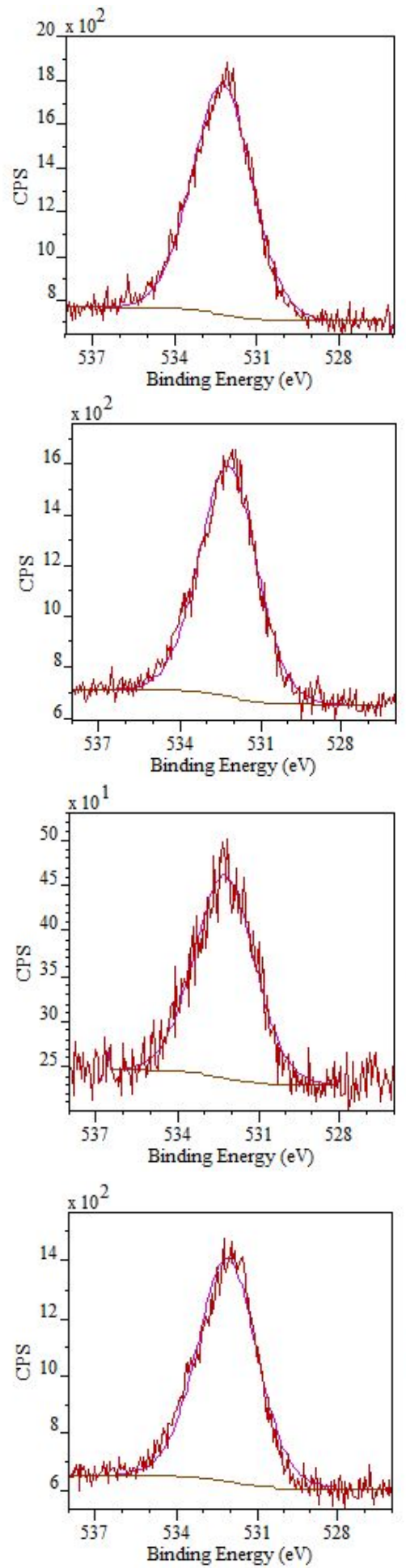

N 1 s
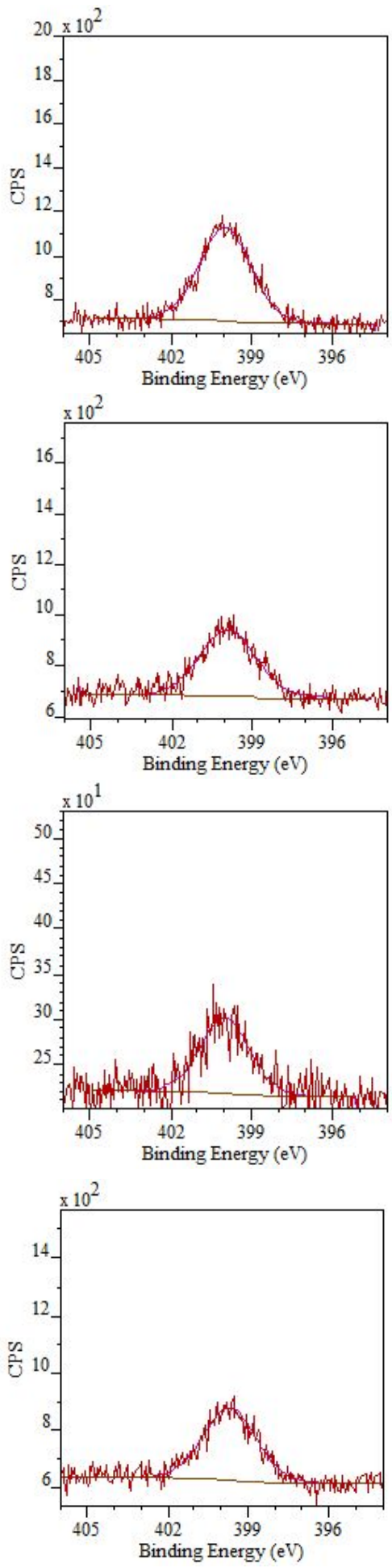

Figure S6. High resolution XPS spectra of $\mathrm{C} 1 s, \mathrm{O} 1 s$, and $\mathrm{N} 1 s$ with individual fitting species for carbon fibers treated at the mixed electrode polarity, $t_{\text {anode }}=5 \mathrm{~min}$. followed by $t_{\text {cathode }}=5 \mathrm{~min}$. $\left(16 \mathrm{~V}, \mathrm{~T}_{i} \approx 23^{\circ} \mathrm{C}\right.$, $0.5 \mathrm{M} \mathrm{NH}_{4} \mathrm{HCO}_{3}$ ). (Spectra corresponds to Table S7. For $\mathrm{C} 1$ s spectra, magenta represents $\mathrm{C}-\mathrm{C} / \mathrm{C}-\mathrm{H}$, aqua blue represents $\mathrm{C}-\mathrm{O} / \mathrm{C}-\mathrm{N} / \mathrm{C}=\mathrm{N}$, red represents $\mathrm{C}=\mathrm{O}$, navy blue represents $\mathrm{O}-\mathrm{C}=\mathrm{O} / \mathrm{N}-\mathrm{C}=\mathrm{O}$ and black represents $\pi \rightarrow \pi^{*}$ shake-up peak.) Surface chemical compositions at $t_{\text {anode }}=5 \mathrm{~min}$. followed by $t_{\text {cathode }}=5 \mathrm{~min}$. are reported in Table S8. 
II e. Fitting of XPS Data: High Resolution XPS Spectra of Carbon Fibers Treated with Ammonium Bicarbonate at Various Initial Temperatures

C $1 s$
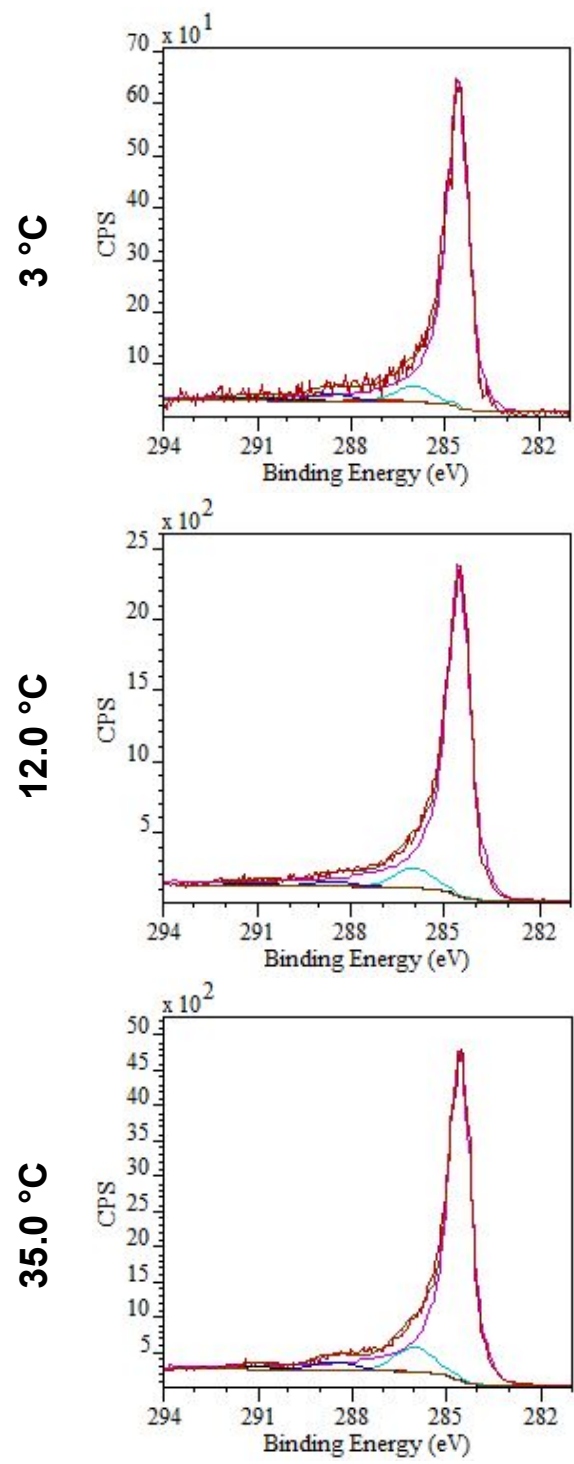

$01 s$
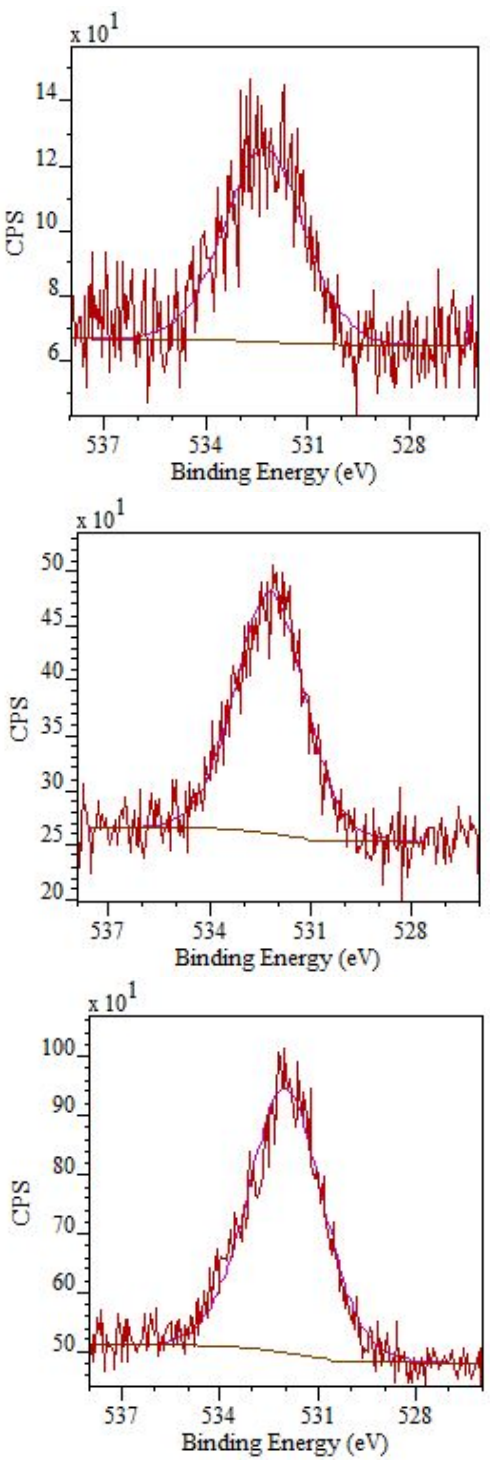

N $1 s$
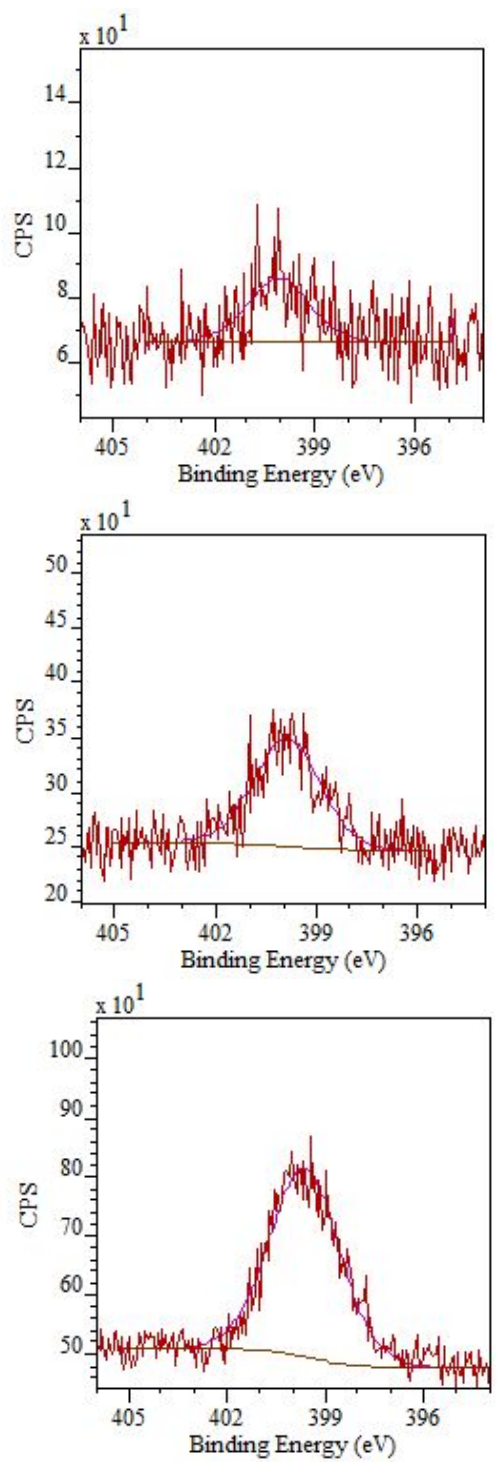

Figure S7. High resolution XPS spectra of $\mathrm{C} 1 s, \mathrm{O} 1 s$, and $\mathrm{N} 1 s$ with individual fitting species for carbon fibers treated at various initial temperatures $\left(16 \mathrm{~V}, t_{\text {anode }}=5 \mathrm{~min}\right.$. followed by $t_{\text {cathode }}=5 \mathrm{~min}$., varying $\mathrm{T}_{i}$, $0.50 \mathrm{M} \mathrm{NH}_{4} \mathrm{HCO}_{3}$ ). (Spectra corresponds to Figure 7 of manuscript. For $\mathrm{C} 1 \mathrm{~s}$ spectra, magenta represents $\mathrm{C}-\mathrm{C} / \mathrm{C}-\mathrm{H}$, aqua blue represents $\mathrm{C}-\mathrm{O} / \mathrm{C}-\mathrm{N} / \mathrm{C}=\mathrm{N}$, red represents $\mathrm{C}=\mathrm{O}$, navy blue represents $\mathrm{O}-\mathrm{C}=\mathrm{O} / \mathrm{N}-\mathrm{C}=\mathrm{O}$ and black represents $\pi \rightarrow \pi^{*}$ shake-up peak.) Surface chemical compositions are reported in Table S9. Spectra for $\mathrm{T}_{i} \approx 23^{\circ} \mathrm{C}$ are reported in Figure S6. 

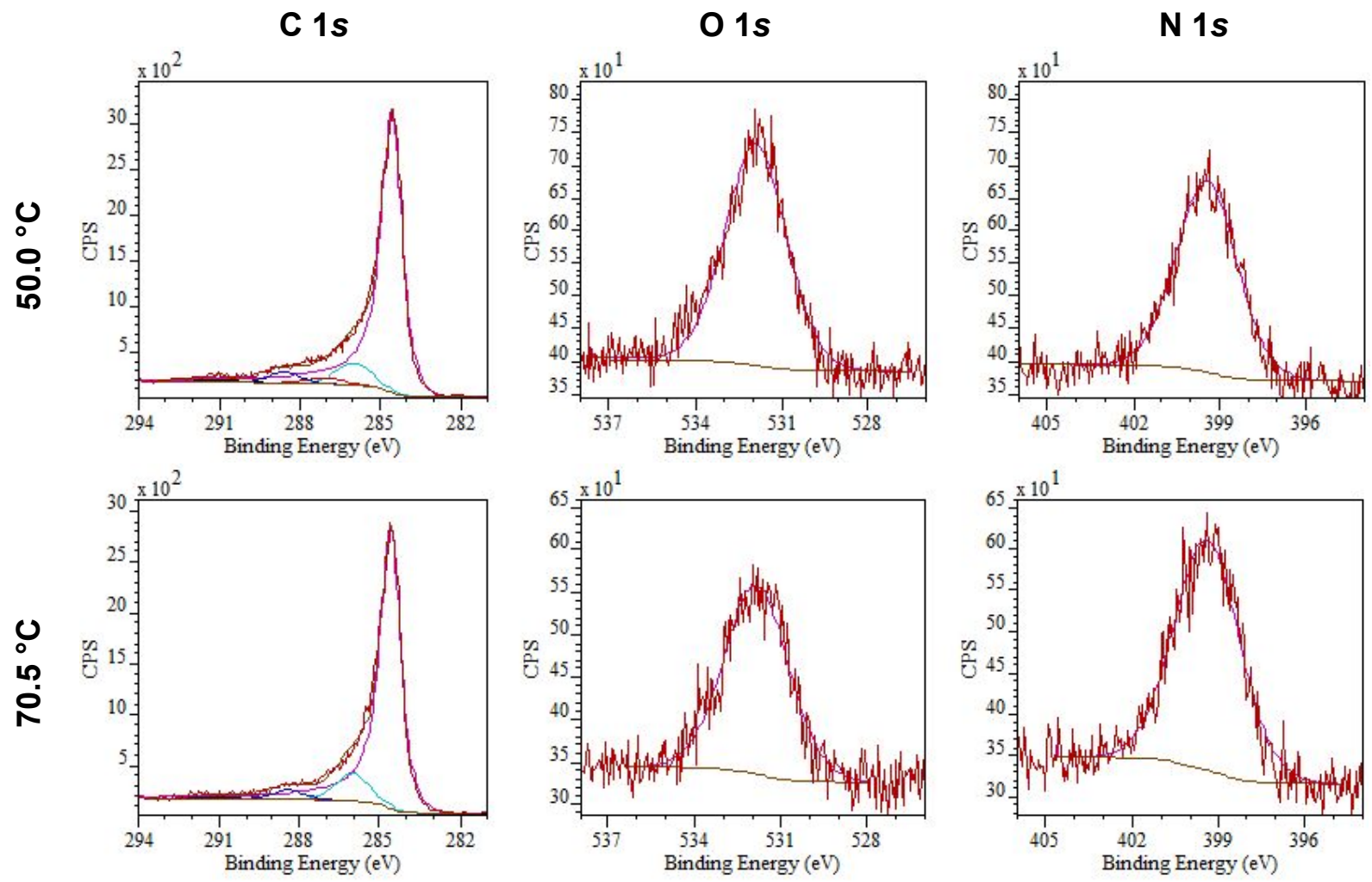

Figure S8. High resolution XPS spectra of $\mathrm{C} 1 \mathrm{~s}, \mathrm{O} 1 \mathrm{~s}$, and $\mathrm{N} 1 \mathrm{~s}$ with individual fitting species for carbon fibers treated at various initial temperatures $\left(16 \mathrm{~V}, t_{\text {anode }}=5 \mathrm{~min}\right.$. followed by $t_{\text {cathode }}=5 \min$., $0.50 \mathrm{M}$ $\mathrm{NH}_{4} \mathrm{HCO}_{3}$ ). (Spectra corresponds to Figure 7 of manuscript. For $\mathrm{C} 1 \mathrm{~s}$ spectra, magenta represents $\mathrm{C}-\mathrm{C} / \mathrm{C}-\mathrm{H}$, aqua blue represents $\mathrm{C}-\mathrm{O} / \mathrm{C}-\mathrm{N} / \mathrm{C}=\mathrm{N}$, red represents $\mathrm{C}=\mathrm{O}$, navy blue represents $\mathrm{O}-\mathrm{C}=\mathrm{O} / \mathrm{N}-\mathrm{C}=\mathrm{O}$ and black represents $\pi \rightarrow \pi^{*}$ shake-up peak.) Surface chemical compositions are reported in Table S9.

Table S9. Surface chemical compositions (at. \%) for the electrolyte concentration studies (Figure 7 of manuscript) based on high resolution XPS analyses. Binding energies (BE) and full width at half maximum $(\mathrm{FWHM})$ are in $\mathrm{eV}$. (16 V, $t_{\text {anode }}=5 \mathrm{~min}$. followed by $t_{\text {cathode }}=5 \mathrm{~min}$., varying $\mathrm{T}_{i}, 0.50 \mathrm{M}$ ammonium bicarbonate)

\begin{tabular}{|c|c|c|c|c|c|c|c|c|c|c|}
\hline \multirow[b]{2}{*}{ element } & \multicolumn{2}{|c|}{$3.0^{\circ} \mathrm{C}$} & \multicolumn{2}{|c|}{$12.0^{\circ} \mathrm{C}$} & \multicolumn{2}{|c|}{$35.0^{\circ} \mathrm{C}$} & \multicolumn{2}{|c|}{$50.0^{\circ} \mathrm{C}$} & \multicolumn{2}{|c|}{$70.5^{\circ} \mathrm{C}$} \\
\hline & $\begin{array}{c}\text { BE } \\
\text { (fwhm) }\end{array}$ & at. $\%$ & $\begin{array}{c}\text { BE } \\
\text { (fwhm) }\end{array}$ & at. $\%$ & $\begin{array}{c}\text { BE } \\
\text { (fwhm) }\end{array}$ & at. $\%$ & $\begin{array}{c}\text { BE } \\
\text { (fwhm) }\end{array}$ & at. $\%$ & $\begin{array}{c}\mathrm{BE} \\
\text { (fwhm) }\end{array}$ & at. $\%$ \\
\hline \multicolumn{11}{|l|}{ C $1 \mathrm{~s}$} \\
\hline $\mathrm{C}-\mathrm{C} / \mathrm{C}-\mathrm{H}$ & $\begin{array}{c}284.6 \\
(0.8)\end{array}$ & $87.2 \pm 1.6$ & $\begin{array}{c}284.6 \\
(0.8)\end{array}$ & $87.6 \pm 0.4$ & $\begin{array}{c}284.6 \\
(0.9)\end{array}$ & $84.9 \pm 0.5$ & $\begin{array}{c}284.6 \\
(0.9)\end{array}$ & $81.8 \pm 0.5$ & $\begin{array}{c}284.6 \\
(0.9)\end{array}$ & $82.8 \pm 1.4$ \\
\hline $\mathrm{C}-\mathrm{O} / \mathrm{C}-\mathrm{N} / \mathrm{C}=\mathrm{N}$ & $\begin{array}{c}286.0 \\
(1.5)\end{array}$ & $7.3 \pm 0.1$ & $\begin{array}{c}286.0 \\
(1.5)\end{array}$ & $7.8 \pm 0.7$ & $\begin{array}{c}286.1 \\
(1.5)\end{array}$ & $9.0 \pm 1.3$ & $\begin{array}{c}286.0 \\
(1.5)\end{array}$ & $9.9 \pm 0.3$ & $\begin{array}{c}286.1 \\
(1.5)\end{array}$ & $10.5 \pm 1.5$ \\
\hline $\mathrm{C}=\mathrm{O} / \mathrm{N}-\mathrm{C}-\mathrm{O}$ & $\begin{array}{r}287.1 \\
(1.5)\end{array}$ & $0.7 \pm 0.5$ & $\begin{array}{c}287.1 \\
(1.5)\end{array}$ & $0.4 \pm 0.2$ & $\begin{array}{c}287.1 \\
(1.5)\end{array}$ & $1.1 \pm 0.5$ & $\begin{array}{c}287.1 \\
(1.5)\end{array}$ & $2.8 \pm 0.2$ & $\begin{array}{c}287.1 \\
(1.5)\end{array}$ & $1.3 \pm 1.3$ \\
\hline $\mathrm{O}-\mathrm{C}=\mathrm{O} / \mathrm{N}-\mathrm{C}=\mathrm{O}$ & $\begin{array}{c}288.6 \\
(1.5)\end{array}$ & $2.9 \pm 0.6$ & $\begin{array}{c}288.6 \\
(1.5)\end{array}$ & $2.9 \pm 0.6$ & $\begin{array}{c}288.6 \\
(1.5)\end{array}$ & $3.8 \pm 0.2$ & $\begin{array}{c}288.6 \\
(1.5)\end{array}$ & $4.4 \pm 0.2$ & $\begin{array}{c}288.6 \\
(1.5)\end{array}$ & $3.8 \pm 0.5$ \\
\hline$\Pi \rightarrow \pi^{*}$ & $\begin{array}{l}291.1 \\
(1.8)\end{array}$ & $2.0 \pm 0.6$ & $\begin{array}{c}291.1 \\
(1.4)\end{array}$ & $1.2 \pm 0.3$ & $\begin{array}{c}291.1 \\
(1.4)\end{array}$ & $1.3 \pm 0.3$ & $\begin{array}{c}291.1 \\
(1.4)\end{array}$ & $1.1 \pm 0.3$ & $\begin{array}{c}291.1 \\
(1.7)\end{array}$ & $1.6 \pm 0.6$ \\
\hline \multicolumn{11}{|l|}{$01 s$} \\
\hline $\mathrm{O}-\mathrm{C} / \mathrm{O}=\mathrm{C}$ & $\begin{array}{c}532.3 \\
(2.8)\end{array}$ & 100 & $\begin{array}{c}532.2 \\
(2.6)\end{array}$ & 100 & $\begin{array}{c}532.0 \\
(2.7)\end{array}$ & 100 & $\begin{array}{l}531.9 \\
(2.6)\end{array}$ & 100 & $\begin{array}{c}531.9 \\
(2.6)\end{array}$ & 100 \\
\hline \multicolumn{11}{|l|}{ N $1 s$} \\
\hline $\mathrm{R}_{2} \mathrm{NH}_{2} / \mathrm{RNH}_{2} / \mathrm{RCONH}_{2}$ & $\begin{array}{c}399.9 \\
(2.4)\end{array}$ & 100 & $\begin{array}{c}399.9 \\
(2.2)\end{array}$ & 100 & $\begin{array}{l}399.6 \\
(2.5)\end{array}$ & 100 & $\begin{array}{c}399.4 \\
(2.5)\end{array}$ & 100 & $\begin{array}{c}399.4 \\
(2.6)\end{array}$ & 100 \\
\hline \multicolumn{11}{|l|}{ others } \\
\hline $\begin{array}{l}\text { Alpha } \\
\text { T range }\left({ }^{\circ} \mathrm{C}\right)\end{array}$ & \multicolumn{2}{|c|}{$\begin{array}{c}0.038 \pm 0.001 \\
3.0-28.0\end{array}$} & \multicolumn{2}{|c|}{$\begin{array}{c}0.039 \pm 0.005 \\
12.0-41.0\end{array}$} & \multicolumn{2}{|c|}{$\begin{array}{c}0.032 \pm 0.003 \\
35.0-69.0\end{array}$} & \multicolumn{2}{|c|}{$\begin{array}{c}0.033 \pm 0.005 \\
50.0-80.0\end{array}$} & \multicolumn{2}{|c|}{$\begin{array}{c}0.030 \pm 0 \\
70.5-84.0\end{array}$} \\
\hline
\end{tabular}


aSurface chemical compositions at room temperature are reported in Table S6.

\section{II f. Fitting of XPS Data: High Resolution XPS Spectra of Carbon Fibers Treated with Varying Ammonium Bicarbonate Concentrations}

C $1 s$
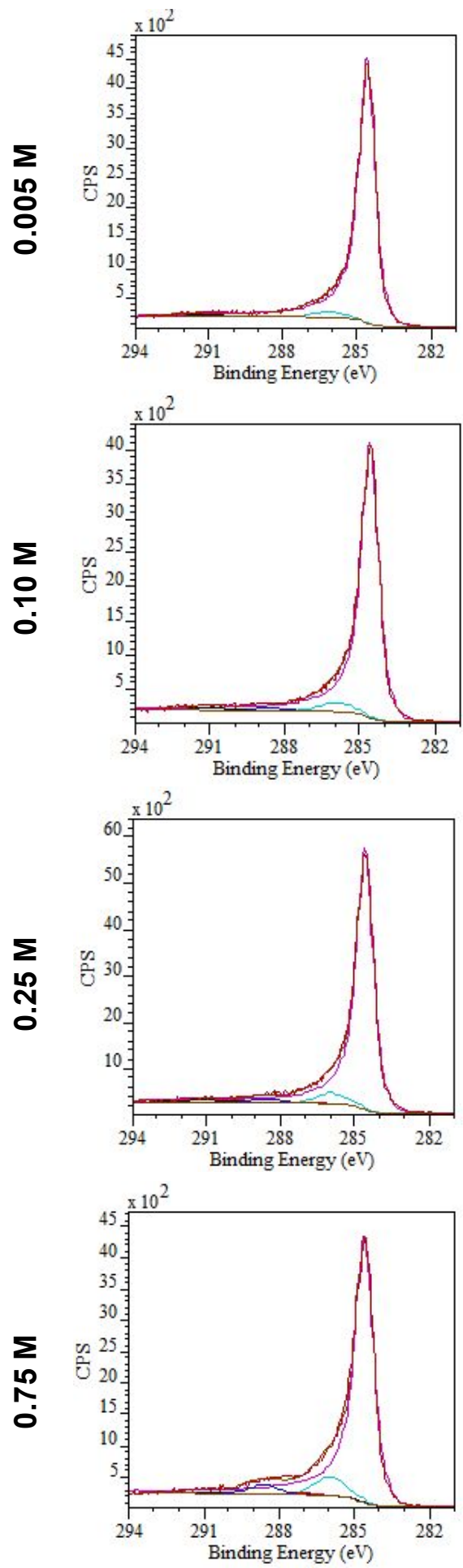

$01 \mathrm{~s}$
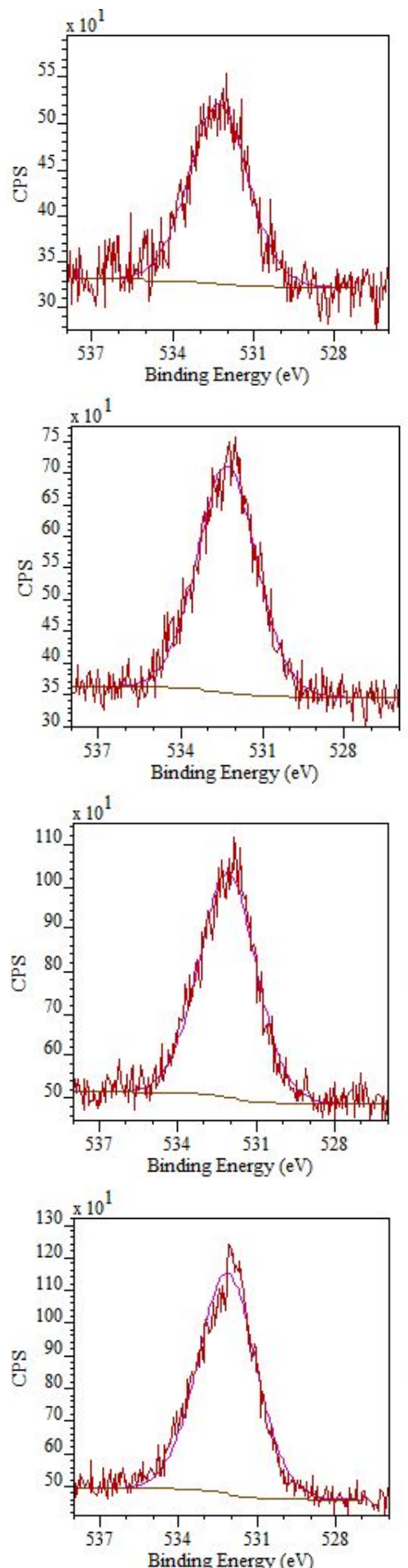

N $1 s$
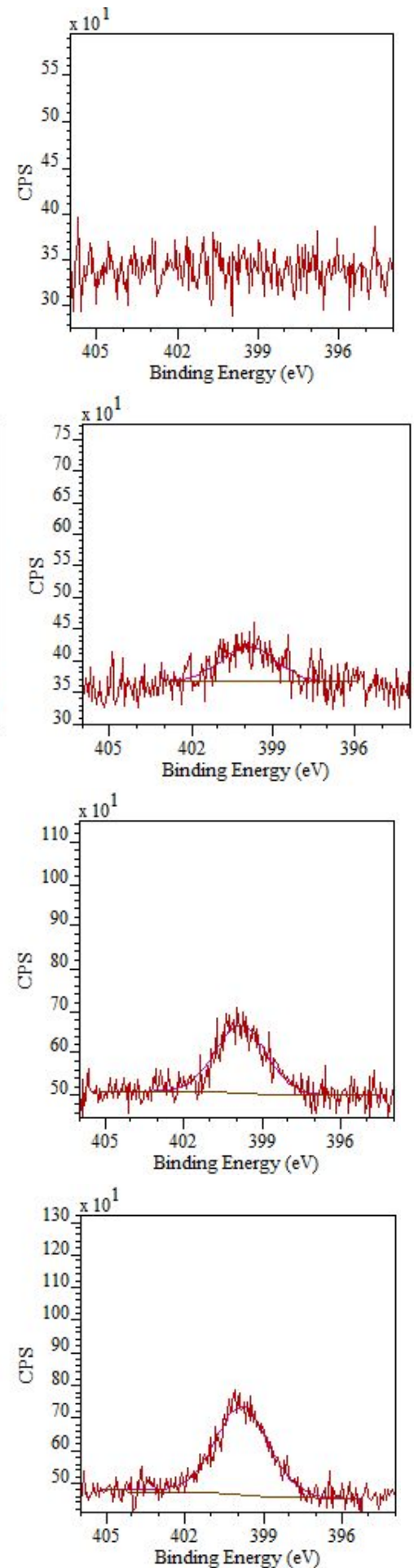

Figure S9. High resolution XPS spectra of $\mathrm{C} 1 \mathrm{~s}, \mathrm{O} 1 \mathrm{~s}$, and $\mathrm{N} 1 \mathrm{~s}$ with individual fitting species for carbon fibers treated at various ammonium bicarbonate concentrations $\left(16 \mathrm{~V}, t_{\text {anode }}=5 \mathrm{~min}\right.$. followed by $t_{\text {cathode }}=5 \mathrm{~min}$., $\mathrm{T}_{i} \approx 23$ ${ }^{\circ} \mathrm{C}$ ). (Spectra corresponds to Figure 7 of manuscript. For $\mathrm{C} 1 \mathrm{~s}$ spectra, magenta represents $\mathrm{C}-\mathrm{C} / \mathrm{C}-\mathrm{H}$, aqua blue 
represents $\mathrm{C}-\mathrm{O} / \mathrm{C}-\mathrm{N} / \mathrm{C}=\mathrm{N}$, red represents $\mathrm{C}=\mathrm{O}$, navy blue represents $\mathrm{O}-\mathrm{C}=\mathrm{O} / \mathrm{N}-\mathrm{C}=\mathrm{O}$ and black represents $\pi \rightarrow \pi^{*}$ shake-up peak.) Spectra for $0.5 \mathrm{M}$ are reported in Figure S6.

Table S8. Surface chemical compositions (at. \%) for the ammonium bicarbonate concentration studies (Figure 8 of manuscript) based on high resolution XPS analyses. Binding energies (BE) and full width at half maximum $(\mathrm{FWHM})$ are in $\mathrm{eV}$. (Applied voltage $=16 \mathrm{~V}, t_{\text {anode }}=5 \mathrm{~min}$. followed by $t_{\text {cathode }}=5 \mathrm{~min}$., $\mathrm{T}_{i} \approx$ $23{ }^{\circ} \mathrm{C}$, varying ammonium bicarbonate concentration)

\begin{tabular}{|c|c|c|c|c|c|c|c|c|}
\hline \multirow[b]{2}{*}{ element } & \multicolumn{2}{|c|}{$0.005 \mathrm{M}$} & \multicolumn{2}{|c|}{$0.10 \mathrm{M}$} & \multicolumn{2}{|c|}{$0.25 \mathrm{M}$} & \multicolumn{2}{|c|}{$0.75 \mathrm{M}$} \\
\hline & $\begin{array}{c}\mathrm{BE} \\
\text { (fwhm) }\end{array}$ & at. $\%$ & $\begin{array}{c}\mathrm{BE} \\
\text { (fwhm) }\end{array}$ & at. $\%$ & $\begin{array}{c}\mathrm{BE} \\
\text { (fwhm) }\end{array}$ & at. $\%$ & $\begin{array}{c}\mathrm{BE} \\
\text { (fwhm) }\end{array}$ & at. $\%$ \\
\hline \multicolumn{9}{|l|}{ C $1 s$} \\
\hline $\mathrm{C}-\mathrm{C} / \mathrm{C}-\mathrm{H}$ & $\begin{array}{l}284.6 \\
(0.8)\end{array}$ & $94.6 \pm 1.0$ & $\begin{array}{l}284.6 \\
(0.8)\end{array}$ & $90.7 \pm 0.4$ & $\begin{array}{c}284.6 \\
(0.8)\end{array}$ & $88.1 \pm 1.5$ & $\begin{array}{l}284.6 \\
(0.9)\end{array}$ & $84.2 \pm 0.5$ \\
\hline $\mathrm{C}-\mathrm{O} / \mathrm{C}-\mathrm{N} / \mathrm{C}=\mathrm{N}$ & $\begin{array}{c}286.0 \\
(1.5)\end{array}$ & $4.2 \pm 0.7$ & $\begin{array}{l}286.0 \\
(1.5)\end{array}$ & $5.7 \pm 1.6$ & $\begin{array}{c}286.1 \\
(1.5)\end{array}$ & $7.0 \pm 0.5$ & $\begin{array}{c}286.0 \\
(1.5)\end{array}$ & $8.6 \pm 0.7$ \\
\hline $\mathrm{C}=\mathrm{O} / \mathrm{N}-\mathrm{C}-\mathrm{O}$ & $\begin{array}{c}287.1 \\
(1.5)\end{array}$ & $0.1 \pm 0.2$ & $\begin{array}{c}287.1 \\
(1.5)\end{array}$ & $0.2 \pm 0.3$ & $\begin{array}{c}287.1 \\
(1.5)\end{array}$ & $1.0 \pm 0.4$ & $\begin{array}{c}287.1 \\
(1.5)\end{array}$ & $1.6 \pm 0.5$ \\
\hline $\mathrm{O}-\mathrm{C}=\mathrm{O} / \mathrm{N}-\mathrm{C}=\mathrm{O}$ & $\begin{array}{l}288.6 \\
(1.5)\end{array}$ & 0 & $\begin{array}{l}288.6 \\
(1.5)\end{array}$ & $1.9 \pm 10.6$ & $\begin{array}{c}288.6 \\
(1.5)\end{array}$ & $2.7 \pm 0.8$ & $\begin{array}{l}288.6 \\
(1.5)\end{array}$ & $4.6 \pm 0.3$ \\
\hline$\Pi \rightarrow \pi^{*}$ & $\begin{array}{l}291.1 \\
(1.2)\end{array}$ & $1.0 \pm 0.6$ & $\begin{array}{l}291.1 \\
(1.4)\end{array}$ & $2.0 \pm 1.8$ & $\begin{array}{c}291.1 \\
(1.8)\end{array}$ & $1.2 \pm 0.6$ & $\begin{array}{l}291.1 \\
(1.3)\end{array}$ & $1.0 \pm 0.5$ \\
\hline \multicolumn{9}{|l|}{$01 \mathrm{~s}$} \\
\hline $\mathrm{O}-\mathrm{C} / \mathrm{O}=\mathrm{C}$ & $\begin{array}{l}532.4 \\
(2.6)\end{array}$ & 100 & $\begin{array}{l}532.2 \\
(2.7)\end{array}$ & 100 & $\begin{array}{l}532.3 \\
(2.7)\end{array}$ & 100 & $\begin{array}{l}532.1 \\
(2.7)\end{array}$ & 100 \\
\hline \multicolumn{9}{|l|}{ N $1 s$} \\
\hline $\mathrm{R}_{2} \mathrm{NH}_{2} / \mathrm{RNH}_{2} / \mathrm{RCONH}_{2}$ & --- & 0 & $\begin{array}{l}399.8 \\
(2.3)\end{array}$ & 100 & $\begin{array}{c}399.9 \\
(2.1)\end{array}$ & 100 & $\begin{array}{l}399.8 \\
(2.3)\end{array}$ & 100 \\
\hline \multicolumn{9}{|l|}{ others } \\
\hline $\begin{array}{l}\text { Alpha } \\
\text { T range }\left({ }^{\circ} \mathrm{C}\right)\end{array}$ & \multicolumn{2}{|c|}{$\begin{array}{c}0.038 \pm 0.010 \\
21.0 \text { (no change) }\end{array}$} & \multicolumn{2}{|c|}{$\begin{array}{c}0.039 \pm 0.005 \\
21.0-34.0\end{array}$} & \multicolumn{2}{|c|}{$\begin{array}{c}0.045 \pm 0.006 \\
21.8-53.0\end{array}$} & \multicolumn{2}{|c|}{$\begin{array}{c}0.045 \pm 0.005 \\
21.0-71.5\end{array}$} \\
\hline
\end{tabular}

aSurface chemical compositions at $0.50 \mathrm{M}$ are reported in Table S7. 


\section{II g. Fitting of XPS Data: High Resolution XPS Spectra of Carbon Fibers Treated with Water and Different Electrolytes in the Mechanism Studies}

C $1 s$
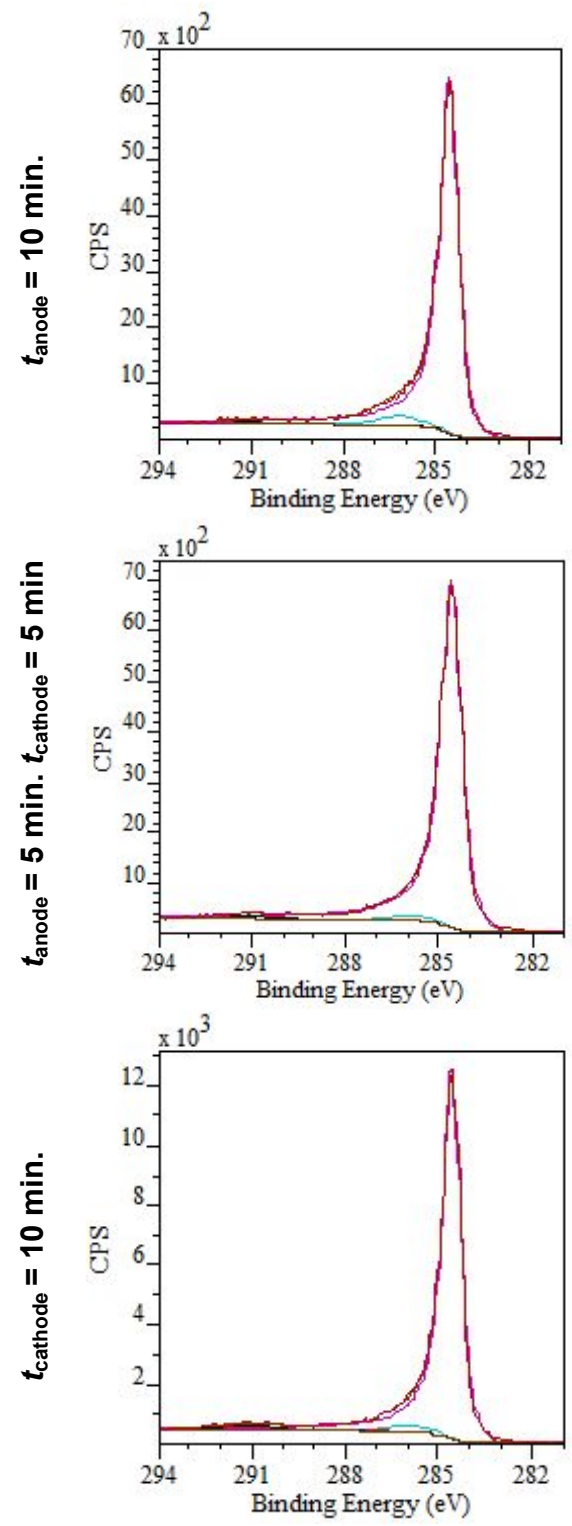

$01 s$
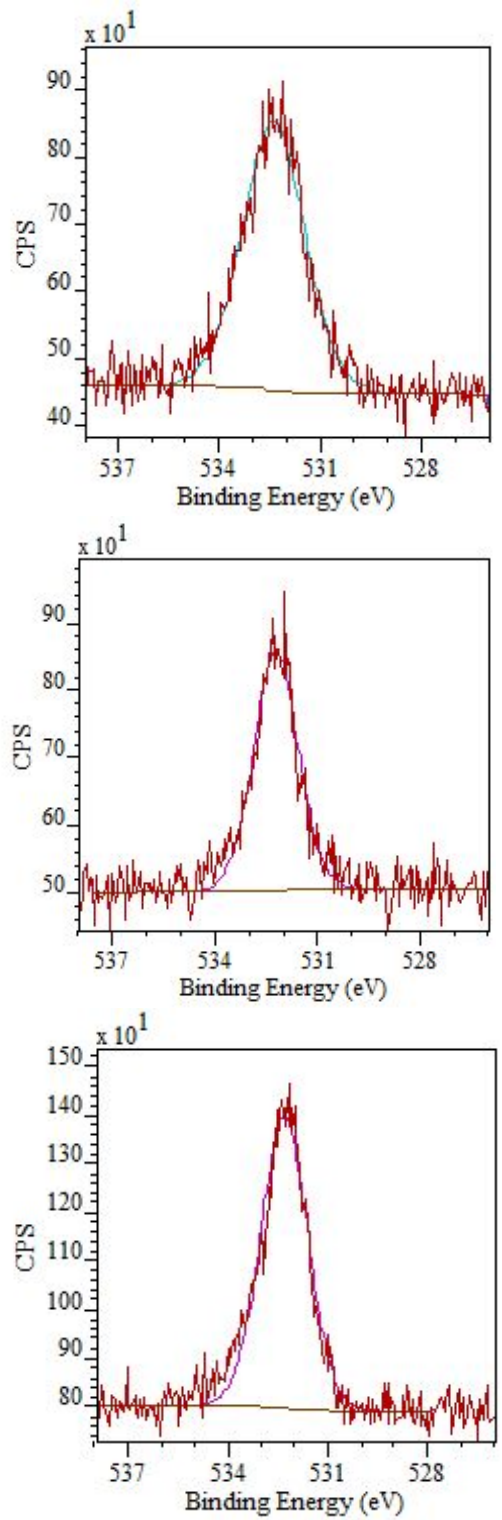

Figure S10. High resolution XPS spectra of $\mathrm{C} 1 \mathrm{~s}, \mathrm{O} 1 \mathrm{~s}$, and $\mathrm{N} 1 \mathrm{~s}$ with individual fitting species for carbon fibers treated with water-only electrolysis $\left(16 \mathrm{~V}\right.$ and $\mathrm{T}_{i} \approx 23^{\circ} \mathrm{C}$ ). (Spectra corresponds to Tables 3-6 of manuscript.) For $\mathrm{C} 1 \mathrm{~s}$ spectra, magenta represents $\mathrm{C}-\mathrm{C} / \mathrm{C}-\mathrm{H}$, aqua blue represents $\mathrm{C}-\mathrm{O} / \mathrm{C}-\mathrm{N} / \mathrm{C}=\mathrm{N}$, red represents $\mathrm{C}=\mathrm{O}$, navy blue represents $\mathrm{O}-\mathrm{C}=\mathrm{O} / \mathrm{N}-\mathrm{C}=\mathrm{O}$ and black represents $\pi \rightarrow \pi^{*}$ shake-up peak.) 
C $1 s$
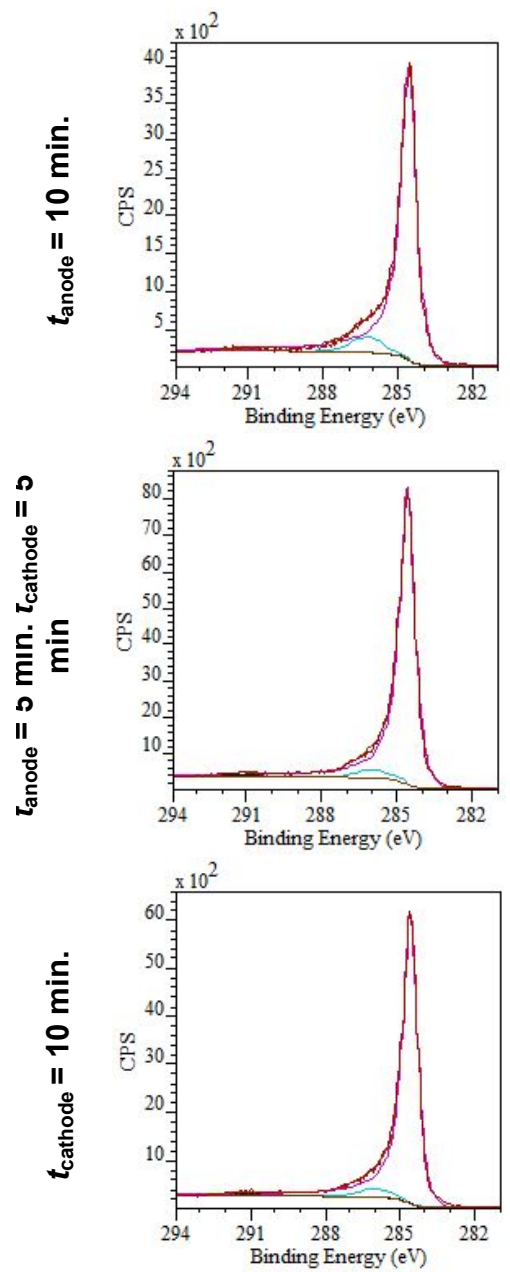

$01 s$
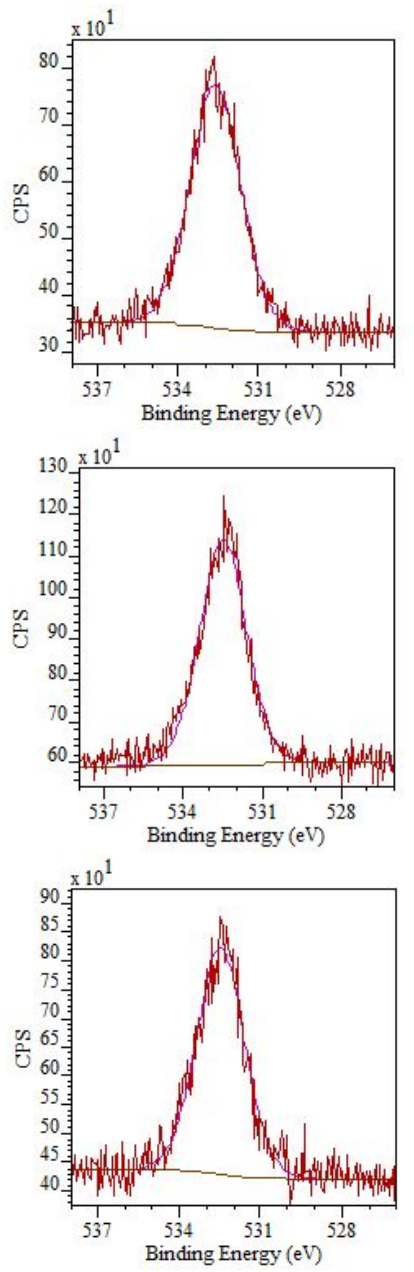

N $1 s$
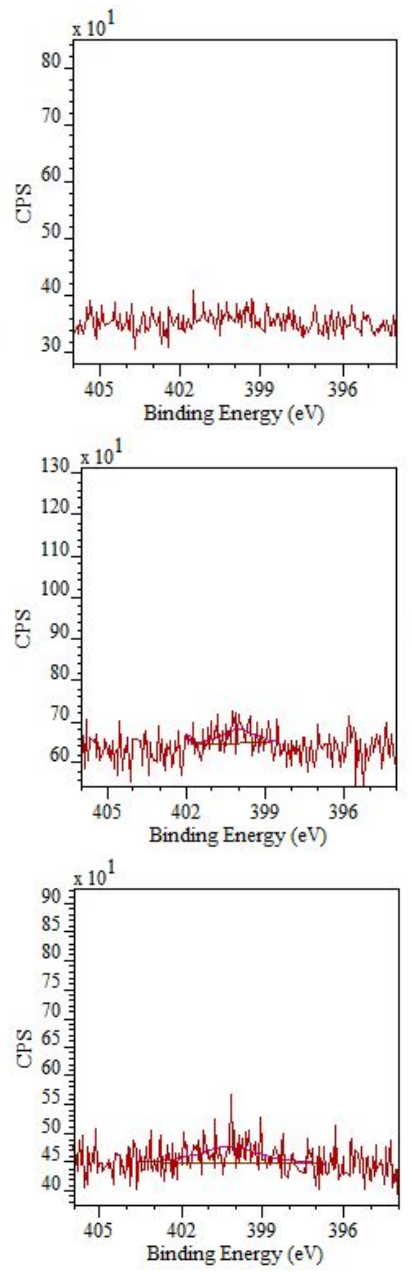

$\mathrm{Cl} 2 p$
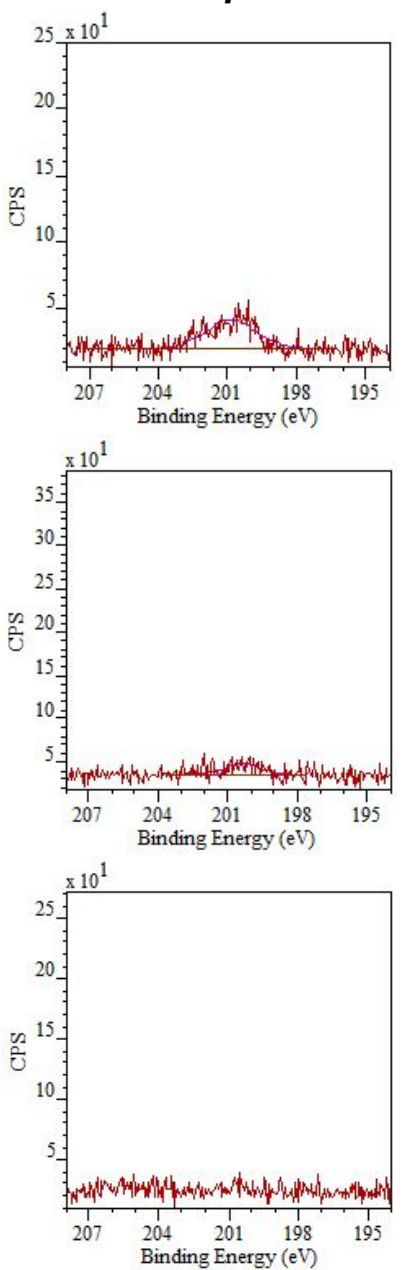

Figure S11. High resolution XPS spectra of $\mathrm{C} 1 s, \mathrm{O} 1 s, \mathrm{~N} 1 s$, and $\mathrm{Cl} 2 p$ with individual fitting species for carbon fibers treated in $0.5 \mathrm{M}$ ammonium chloride $\left(16 \mathrm{~V}\right.$ and $\mathrm{T}_{i} \approx 23^{\circ} \mathrm{C}$ ). (Spectra corresponds to Tables 3-6 of manuscript.) For $\mathrm{C}$ 1s spectra, magenta represents $\mathrm{C}-\mathrm{C} / \mathrm{C}-\mathrm{H}$, aqua blue represents $\mathrm{C}-\mathrm{O} / \mathrm{C}-\mathrm{N} / \mathrm{C}=\mathrm{N}$, red represents $\mathrm{C}=\mathrm{O}$, navy blue represents $\mathrm{O}-\mathrm{C}=\mathrm{O} / \mathrm{N}-\mathrm{C}=\mathrm{O}$ and black represents $\pi \rightarrow \pi^{*}$ shake-up peak.) 
C 1 s
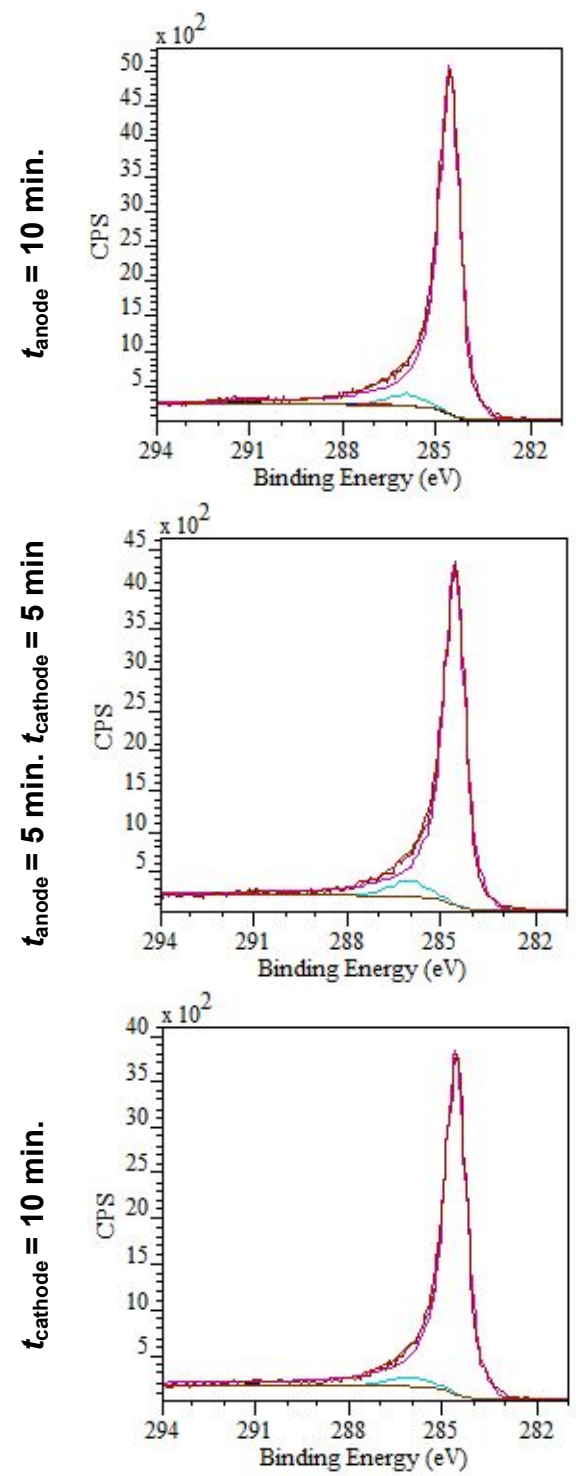

$01 s$
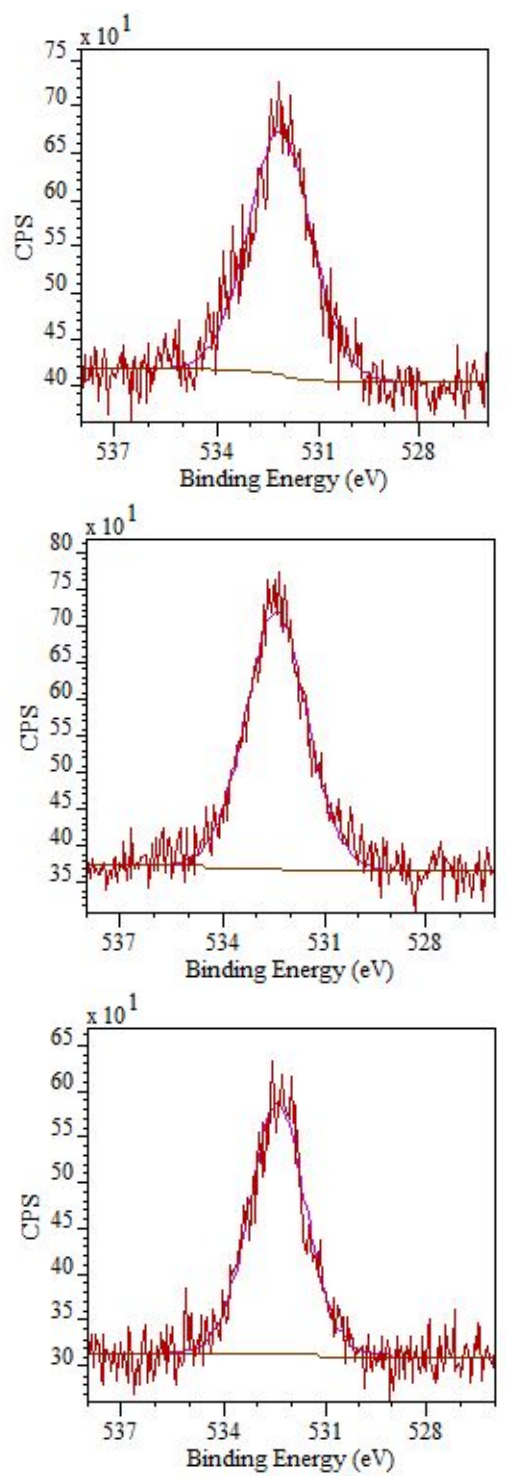

N $1 s$
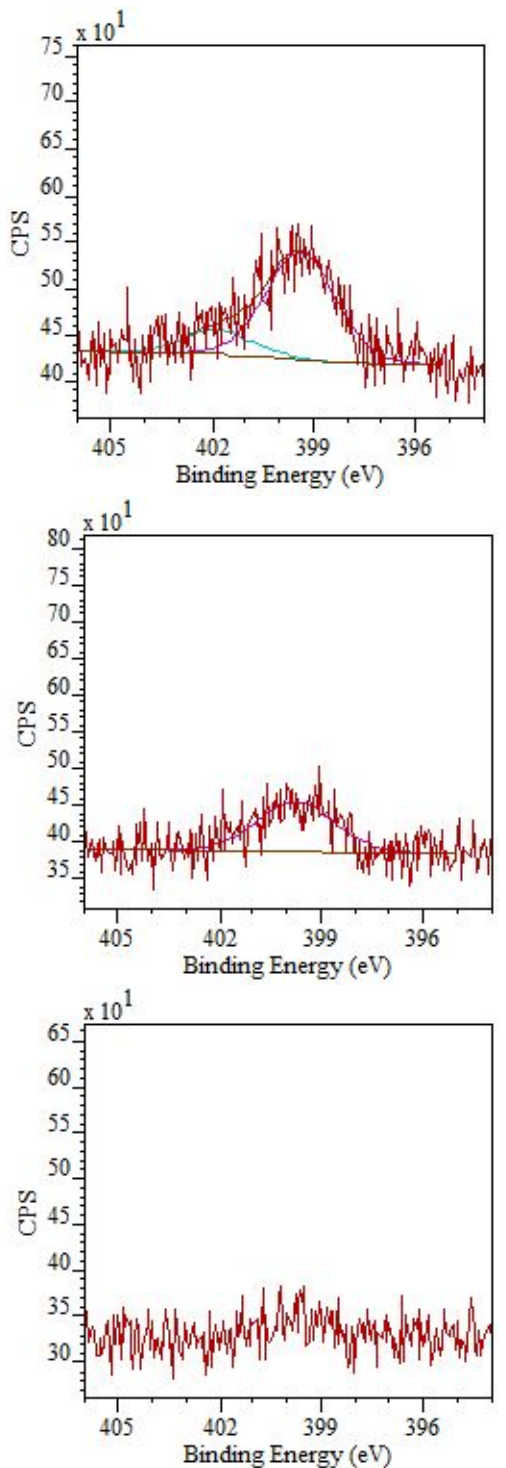

Figure S12. High resolution XPS spectra of $\mathrm{C} 1 s, \mathrm{O} 1 s$, and $\mathrm{N} 1 s$ with individual fitting species for carbon fibers treated in $0.5 \mathrm{M}$ ammonium hydroxide $\left(16 \mathrm{~V}\right.$ and $\mathrm{T}_{i} \approx 23^{\circ} \mathrm{C}$ ). (Spectra corresponds to Tables 3-6 of manuscript.) For $\mathrm{C} 1 \mathrm{~s}$ spectra, magenta represents $\mathrm{C}-\mathrm{C} / \mathrm{C}-\mathrm{H}$, aqua blue represents $\mathrm{C}-\mathrm{O} / \mathrm{C}-\mathrm{N} / \mathrm{C}=\mathrm{N}$, red represents $\mathrm{C}=\mathrm{O}$, navy blue represents $\mathrm{O}-\mathrm{C}=\mathrm{O} / \mathrm{N}-\mathrm{C}=\mathrm{O}$ and black represents $\pi \rightarrow \pi^{*}$ shake-up peak. For $\mathrm{N} 1 s$ spectra, magenta represents signal at $\sim 399.5 \mathrm{eV}$ and aqua blue represents $\mathrm{RNH}_{3}{ }^{+}$at $\sim 401.8$ $\mathrm{eV}$.) 
C $1 s$
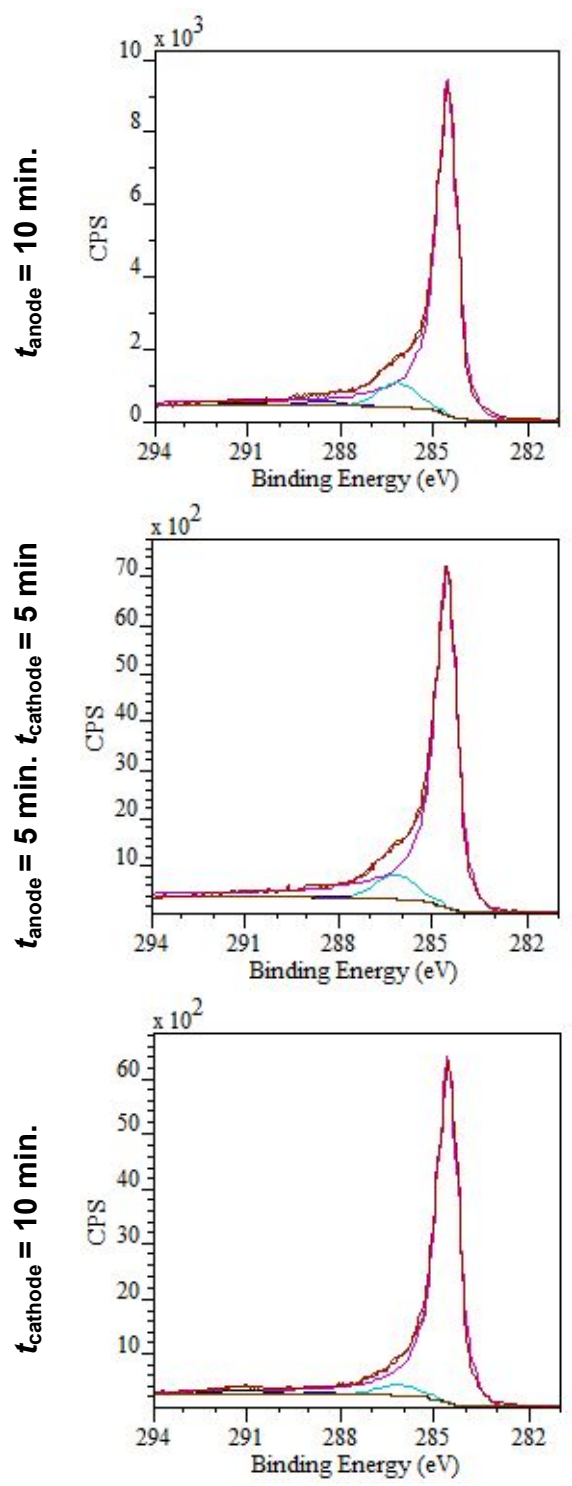

$01 s$
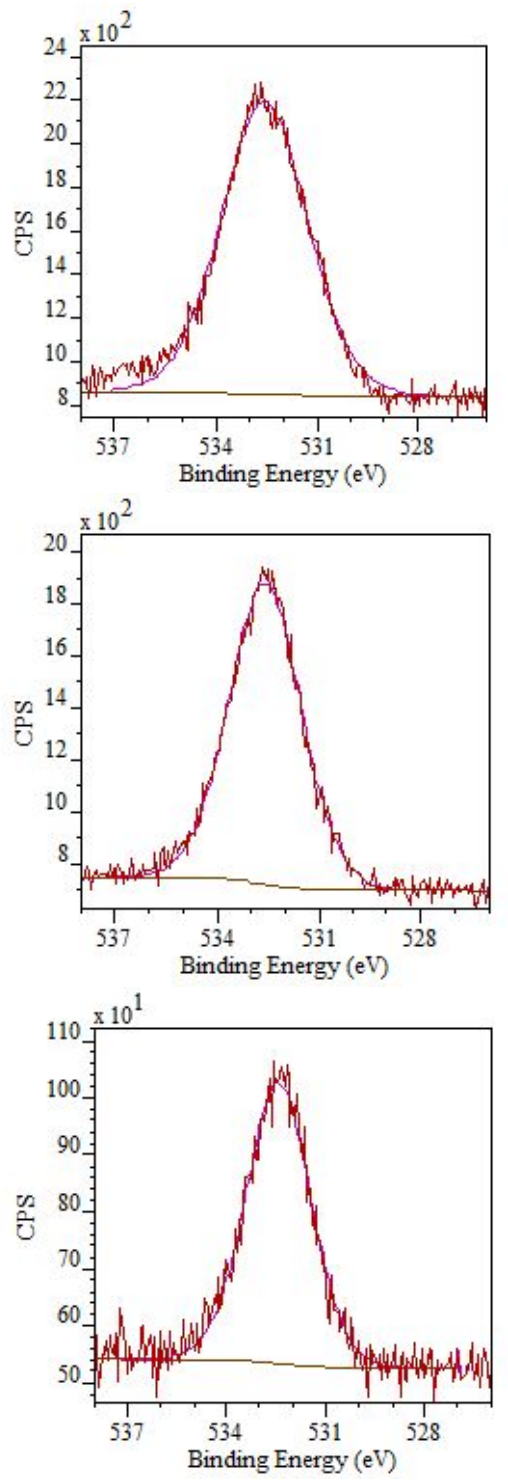

$\mathrm{Na} 1 \mathrm{~s}$
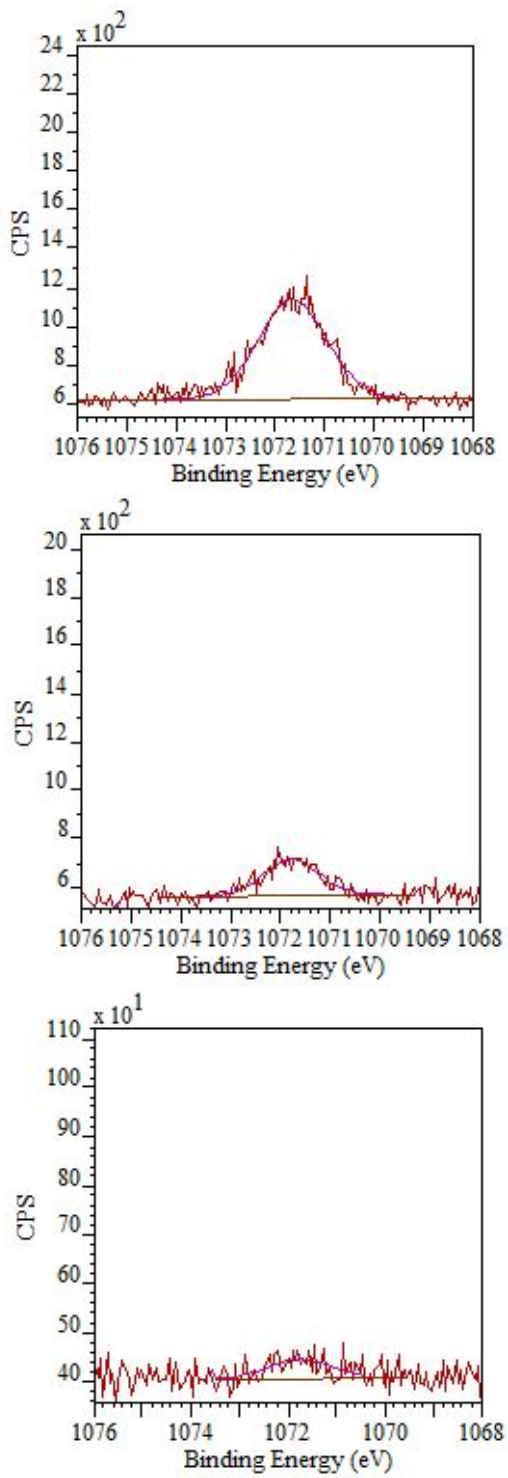

Figure S13. High resolution XPS spectra of $\mathrm{C} 1 \mathrm{~s}, \mathrm{O} 1 \mathrm{~s}$, and $\mathrm{N} 1 \mathrm{~s}$ with individual fitting species for carbon fibers treated in $0.5 \mathrm{M}$ sodium bicarbonate $\left(16 \mathrm{~V}\right.$ and $\left.\mathrm{T}_{i} \approx 23^{\circ} \mathrm{C}\right)$. (Spectra corresponds to Tables $3-6$ of manuscript). For $\mathrm{C} 1 \mathrm{~s}$ spectra, magenta represents $\mathrm{C}-\mathrm{C} / \mathrm{C}-\mathrm{H}$, aqua blue represents $\mathrm{C}-\mathrm{O} / \mathrm{C}-\mathrm{N} / \mathrm{C}=\mathrm{N}$, red represents $\mathrm{C}=\mathrm{O}$, navy blue represents $\mathrm{O}-\mathrm{C}=\mathrm{O} / \mathrm{N}-\mathrm{C}=\mathrm{O}$ and black represents $\pi \rightarrow \pi^{*}$ shake-up peak.) 


\section{C $1 s$}
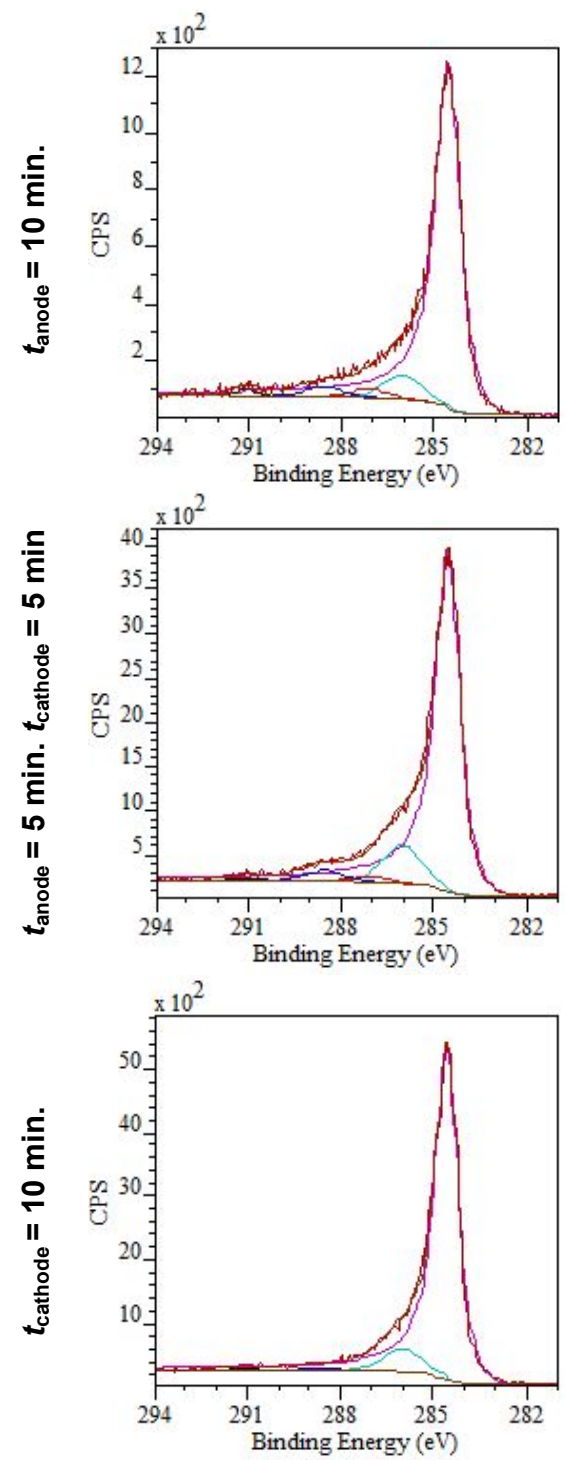

$01 s$
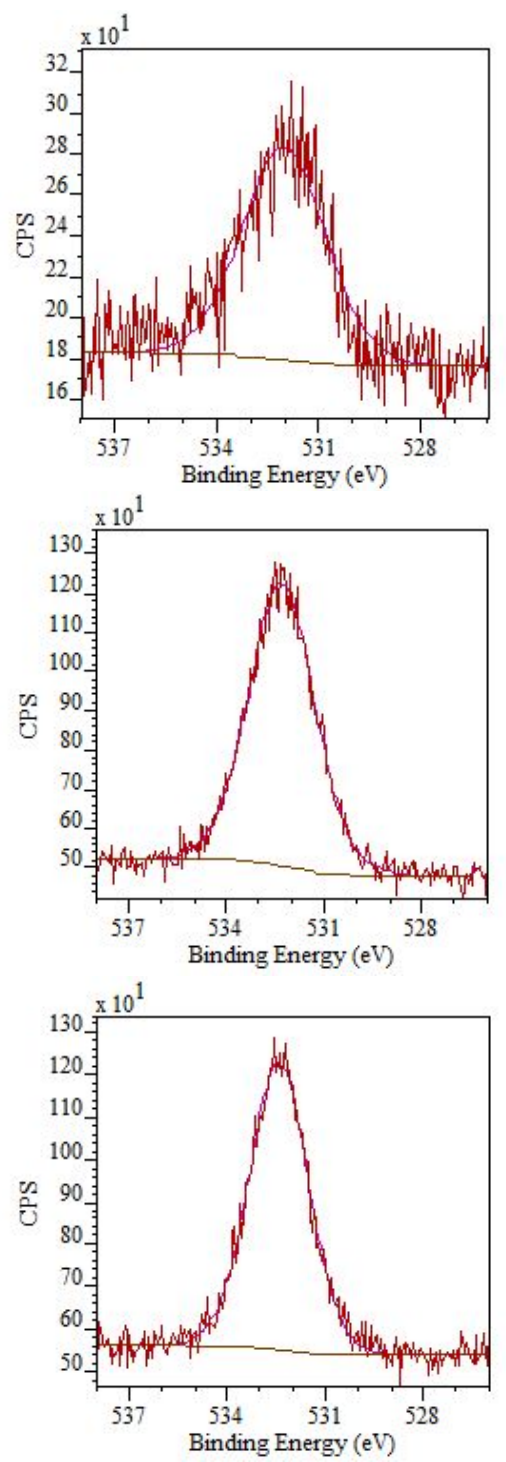

N $1 s$
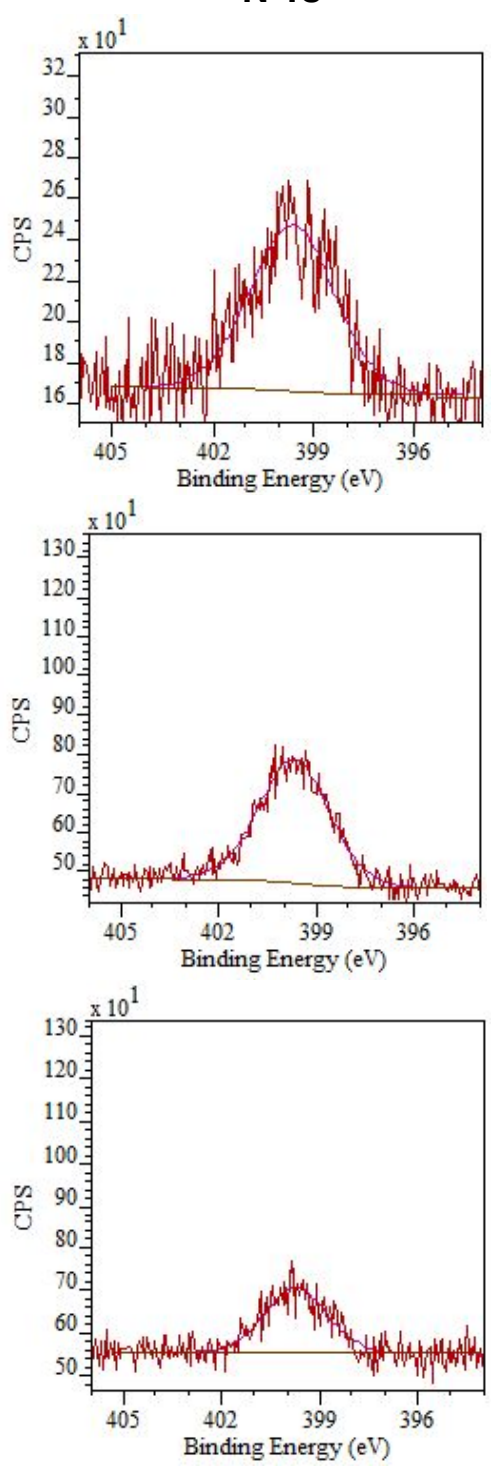

Figure S14. High resolution XPS spectra of $\mathrm{C} 1 s, \mathrm{O} 1 s$, and $\mathrm{N} 1 s$ with individual fitting species for carbon fibers treated in $0.5 \mathrm{M}$ ammonium carbamate $\left(16 \mathrm{~V}\right.$ and $\mathrm{T}_{i} \approx 23^{\circ} \mathrm{C}$ ). (Spectra corresponds to Tables $3-6$ of manuscript). For $\mathrm{C} 1 \mathrm{~s}$ spectra, magenta represents $\mathrm{C}-\mathrm{C} / \mathrm{C}-\mathrm{H}$, aqua blue represents $\mathrm{C}-\mathrm{O} / \mathrm{C}-\mathrm{N} / \mathrm{C}=\mathrm{N}$, red represents $\mathrm{C}=\mathrm{O}$, navy blue represents $\mathrm{O}-\mathrm{C}=\mathrm{O} / \mathrm{N}-\mathrm{C}=\mathrm{O}$ and black represents $\pi \rightarrow \pi^{*}$ shake-up peak.) 


\section{References}

[1] H. Min, P.-L. Girard-Lauriault, T. Gross, A. Lippitz, P. Dietrich, and W. E. S. Unger, "Ambient-ageing processes in amine self-assembled monolayers on microarray slides as studied by ToF-SIMS with principal component analysis, XPS, and NEXAFS spectroscopy," Analytical and Bioanalytical Chemistry, vol. 403, pp. 613-623, 2012.

[2] R. G. Acres, A. V. Ellis, J. Alvino, C. E. Lenahan, D. A. Khodakov, G. F. Metha, et al., "Molecular Structure of 3Aminopropyltriethoxysilane Layers Formed on Silanol-Terminated Silicon Surfaces," The Journal of Physical Chemistry C, vol. 116, pp. 6289-6297, 2012/03/15 2012.

[3] Y. Ding, L.-T. Weng, M. Yang, Z. Yang, X. Lu, N. Huang, et al., "Insights into the Aggregation/Deposition and Structure of a Polydopamine Film," Langmuir, vol. 30, pp. 12258-12269, 2014/10/21 2014.

[4] R. A. Zangmeister, T. A. Morris, and M. J. Tarlov, "Characterization of Polydopamine Thin Films Deposited at Short Times by Autoxidation of Dopamine," Langmuir, vol. 29, pp. 8619-8628, Jul 92013.

[5] K. Shimizu, C. Phanopoulos, R. Loenders, M.-L. Abel, and J. F. Watts, "The characterization of the interfacial interaction between polymeric methylene diphenyl diisocyanate and aluminum: a ToF-SIMS and XPS study," Surface and Interface Analysis, vol. 42, pp. 1432-1444, 2010.

[6] M. B. Clark, J. A. Gardella, T. M. Schultz, D. G. Patil, and L. Salvati, "Solid-state analysis of eumelanin biopolymers by electron spectroscopy for chemical analysis," Analytical Chemistry, vol. 62, pp. 949-956, 1990/05/01 1990.

[7] A. K. Chauhan, D. K. Aswal, S. P. Koiry, S. K. Gupta, J. V. Yakhmi, C. Sürgers, et al., "Self-assembly of the 3aminopropyltrimethoxysilane multilayers on Si and hysteretic current-voltage characteristics," Applied Physics A, vol. 90, pp. 581-589, 2008.

[8] B. Kannan, D. A. Higgins, and M. M. Collinson, "Aminoalkoxysilane Reactivity in Surface Amine Gradients Prepared by Controlled-Rate Infusion," Langmuir, vol. 28, pp. 16091-16098, 2012/11/20 2012.

[9] H. J. Martin, K. H. Schulz, J. D. Bumgardner, and K. B. Walters, "XPS Study on the Use of 3Aminopropyltriethoxysilane to Bond Chitosan to a Titanium Surface," Langmuir, vol. 23, pp. 6645-6651, 2007/06/01 2007.

[10] H. J. Martin, K. H. Schulz, J. D. Bumgardner, and K. B. Walters, "An XPS study on the attachment of triethoxsilylbutyraldehyde to two titanium surfaces as a way to bond chitosan," Applied Surface Science, vol. 254, pp. 4599-4605, 2008.

[11] R. Schlögl and H. P. Boehm, "Influence of crystalline perfection and surface species on the X-ray photoelectron spectra of natural and synthetic graphites," Carbon, vol. 21, pp. 345-358, 1983/01/01/ 1983.

[12] J. Lahaye, G. Nansé, A. Bagreev, and V. Strelko, "Porous structure and surface chemistry of nitrogen containing carbons from polymers," Carbon, vol. 37, pp. 585-590, 1999/03/08/ 1999.

[13] R. J. J. Jansen and H. van Bekkum, "XPS of nitrogen-containing functional groups on activated carbon," Carbon, vol. 33, pp. 1021-1027, 1995/01/01/ 1995.

[14] B. Stöhr, H. P. Boehm, and R. Schlögl, "Enhancement of the catalytic activity of activated carbons in oxidation reactions by thermal treatment with ammonia or hydrogen cyanide and observation of a superoxide species as a possible intermediate," Carbon, vol. 29, pp. 707-720, 1991/01/01/ 1991.

[15] L. R. Radovic, I. F. Silva, J. I. Ume, J. A. Menéndez, C. A. L. Y. Leon, and A. W. Scaroni, "An experimental and theoretical study of the adsorption of aromatics possessing electron-withdrawing and electron-donating functional groups by chemically modified activated carbons," Carbon, vol. 35, pp. 1339-1348, 1997/01/01/ 1997.

[16] B. J. Meldrum and C. H. Rochester, "In situ infrared study of the modification of the surface of activated carbon by ammonia, water and hydrogen," Journal of the Chemical Society, Faraday Transactions, vol. 86, pp. 1881-1884, 1990.

[17] NIST website: https://srdata.nist.gov/xps/Default.aspx 